\title{
Human neutralizing antibodies elicited by SARS-CoV-2 infection
}

https://doi.org/10.1038/s41586-020-2380-z

Received: 13 March 2020

Accepted: 18 May 2020

Published online: 26 May 2020

Check for updates

\begin{abstract}
Bin Ju,2,9 , Qi Zhang ${ }^{3,9}$, Jiwan $\mathrm{Ge}^{4,9}$, Ruoke Wang ${ }^{3}$, Jing Sun ${ }^{5}$, Xiangyang $\mathrm{Ge}^{1}$, Jiazhen $\mathrm{Yu}^{1}$, Sisi Shan ${ }^{3}$, Bing Zhou', Shuo Song ', Xian Tang', Jinfang Yu ${ }^{4}$, Jun Lan ${ }^{4}$, Jing Yuan ${ }^{6}$, Haiyan Wang', Juanjuan Zhao ${ }^{1,2}$, Shuye Zhang ${ }^{7}$, Youchun Wang ${ }^{8}$, Xuanling Shi ${ }^{3}$, Lei Liu ${ }^{1,2}$, Jincun Zhao ${ }^{5}$, Xinquan Wang ${ }^{4 凶}$, Zheng Zhang ${ }^{1,2 \bowtie}$ \& Linqi Zhang ${ }^{3 凶}$
\end{abstract}

\begin{abstract}
The coronavirus disease 2019 (COVID-19) pandemic caused by severe acute respiratory syndrome coronavirus 2 (SARS-CoV-2) presents a global health emergency that is in urgent need of intervention ${ }^{1-3}$. The entry of SARS-CoV-2 into its target cells depends on binding between the receptor-binding domain (RBD) of the viral spike protein and its cellular receptor, angiotensin-converting enzyme 2 $(\text { ACE2 })^{2,4-6}$. Here we report the isolation and characterization of 206 RBD-specific monoclonal antibodies derived from single B cells from 8 individuals infected with SARS-CoV-2. We identified antibodies that potently neutralize SARS-CoV-2; this activity correlates with competition with ACE2 for binding to RBD. Unexpectedly, the anti-SARS-CoV-2 antibodies and the infected plasma did not cross-react with the RBDs of SARS-CoV or Middle East respiratory syndrome-related coronavirus (MERS-CoV), although there was substantial plasma cross-reactivity to their trimeric spike proteins. Analysis of the crystal structure of RBD-bound antibody revealed that steric hindrance inhibits viral engagement with ACE2, thereby blocking viral entry. These findings suggest that anti-RBD antibodies are largely viral-species-specific inhibitors. The antibodies identified here may be candidates for development of clinical interventions against SARS-CoV-2.
\end{abstract}

The rapid international transmission of SARS-CoV-2 poses a serious global health emergency with no available treatments or vaccine $\mathrm{e}^{1-3}$. SARS-CoV-2 shares substantial genetic and functional similarity with other human betacoronaviruses, including SARS-CoV and MERS-CoV ${ }^{2,4-8}$.

SARS-CoV-2 uses an envelope homotrimeric spike glycoprotein to interact with the cellular receptor ACE2 ${ }^{2,5,6,8}$. Binding with ACE2 triggers a cell membrane fusion cascade that results in viral entry. This suggests that disruption of the RBD-ACE2 interaction would block SARS-CoV-2 cell entry. The high-resolution structure of SARS-CoV-2 RBD bound to the $\mathrm{N}$-terminal peptidase domain of ACE2 has recently been determined $^{6-8}$. The ACE2-binding mechanism is nearly identical between SARS-CoV-2 and SARS-CoV RBDs ${ }^{7-10}$. Animal studies on RBD-based vaccines against SARS-CoV and MERS-CoV have shown strong polyclonal antibody responses that inhibit viral entry ${ }^{11,12}$. These findings suggest that anti-RBD antibodies should effectively block SARS-CoV-2 entry. In this study, we report on RBD-specific monoclonal antibodies obtained from individuals infected with SARS-CoV-2.

\section{Plasma antibody response against SARS-CoV-2}

We collected cross-sectional and longitudinal blood samples from eight patients infected with SARS-CoV-2, who were infected during the early outbreak in Shenzhen (Supplementary Table 1). Samples were named according to patient ID and A, B, or C depending on when they were collected. Six patients ( $\mathrm{P} 1$ to $\mathrm{P} 4, \mathrm{P} 8$ and $\mathrm{P} 16$ ) had recently travelled to Wuhan and the others (P5 and P22) had direct contact with people who had recently been in Wuhan. P1 to P5 comprise a family cluster, including the first documented case of human-to-human transmission in Shenzhen ${ }^{13}$. All patients recovered except for $\mathrm{P} 1$, who succumbed to COVID-19. Antibody binding was quantified using serial plasma dilutions on enzyme-linked immunosorbent assay (ELISA) plates coated with either recombinant RBD, trimeric spike protein derived from SARS-CoV-2, SARS-CoV or MERS-CoV, or recombinant nucleoprotein from SARS-CoV-2. Binding activity was visualized by adding anti-human IgG antibodies and measuring absorbance at $450 \mathrm{~nm}\left(A_{450}\right)$. Samples from P1, P2, P5 and P16 showed increased binding to both SARS-CoV-2 RBD and nucleoprotein compared

${ }^{1}$ Institute for Hepatology, National Clinical Research Center for Infectious Disease, Shenzhen Third People's Hospital, Shenzhen, China. ${ }^{2}$ The Second Affiliated Hospital, School of Medicine,

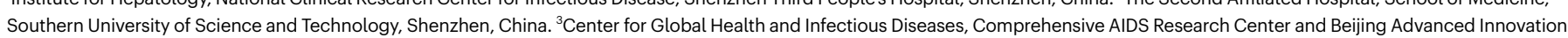

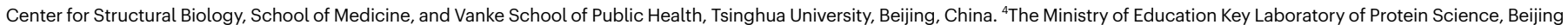

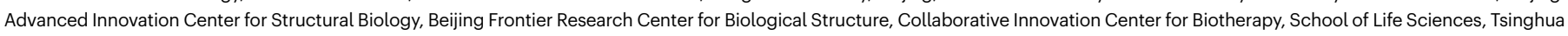

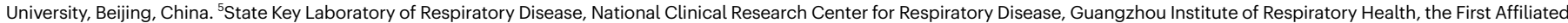
Hospital of Guangzhou Medical University, Guangzhou, China. ${ }^{6}$ Department for Infectious Diseases, Shenzhen Third People's Hospital, Shenzhen, China. ${ }^{7}$ Shanghai Public Health Clinical

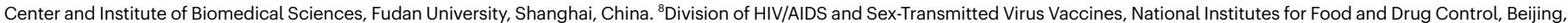
China. ${ }^{9}$ These authors contributed equally: Bin Ju, Qi Zhang, Jiwan Ge. ${ }^{凶}$ e-mail: xinquanwang@mail.tsinghua.edu.cn; zhangzheng1975@aliyun.com; zhanglinqi@tsinghua.edu.cn 

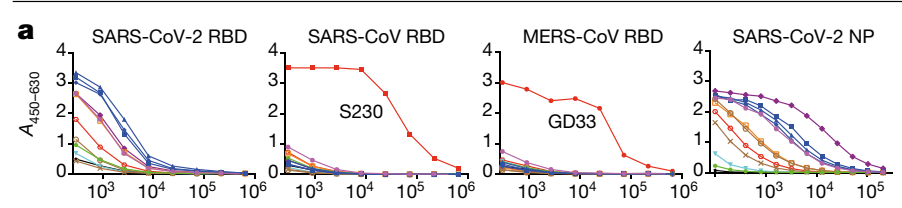

b SARS-CoV-2 Spike
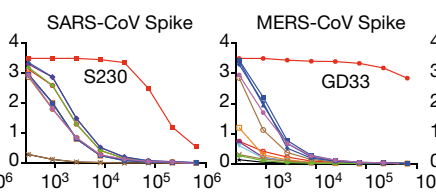

HIV-1 BG505 Trimer
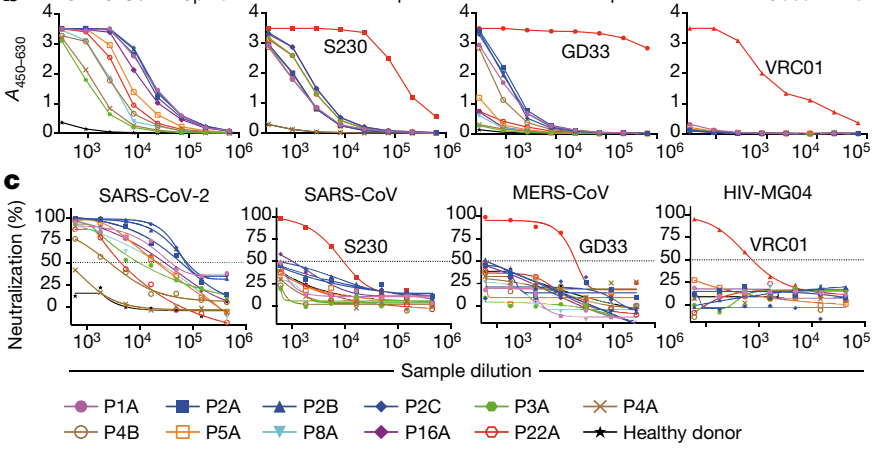

Fig. 1 Analyses of plasma response to SARS-CoV-2.a-c, Serial dilutions of plasma samples were analysed for binding to RBDs (and SARS-CoV-2 nucleoprotein (NP)) (a) and trimeric spikes (b) of SARS-CoV-2, SARS-CoV and MERS-CoV by ELISA, or for neutralization against pseudoviruses bearing envelope glycoprotein of SARS-CoV-2, SARS-CoV and MERS-CoV (c). Positive control monoclonal antibodies for SARS-CoV (S230), MERS-CoV (GD33) and HIV-1 (VRC01) were included throughout. Results are representatives of two independent experiments.

with the other samples (Fig. 1a). Three sequential plasma samples showed relatively stable binding over the sampling period. We found no cross-reactivity with SARS-CoV and MERS-CoV RBDs (Fig. 1a). However, strong cross-reactivity was detected in ELISA (Fig. 1b) and cell-surface staining (Extended Data Fig. 1) using trimeric spike protein from SARS-CoV and MERS-CoV. All samples except those from P4A exhibited binding to SARS-CoV trimeric spike, whereas P1, P2 and $\mathrm{P} 4 \mathrm{~B}$ samples recognized MERS-CoV trimeric spike (Fig. 1b). No samples reacted with the control BG505-derived human immunodeficiency virus-1 (HIV-1) envelope trimer ${ }^{14}$. SARS-CoV-2-neutralizing activities varied across patients (Fig. 1c), consistent with variation in binding activity. Cross-neutralization against SARS-CoV and MERS-CoV was negligible for all plasma samples (Fig. 1c). These results suggest that SARS-CoV-2, SARS-CoV and MERS-CoV RBDs are immunologically distinct and that non-RBD regions contribute to the observed cross-reactivity with SARS-CoV and MERS-CoV spike protein.

\section{Single $B$ cell antibody cloning and analysis}

Flow cytometry was used to isolate $B$ cells that recognize fluorescent RBD probes. The proportions of RBD-binding B cells varied among the patient samples, but were significantly higher than those from uninfected control individuals (Fig. 2a and Extended Data Fig. 2). Those from P2, P5, P16 and P22 were particularly high, consistent with their level of plasma binding to SARS-CoV-2 RBD and spike protein (Fig. 1a, b). We isolated and cloned RBD-binding B cells as previously described ${ }^{15}$. In total, 206 of 358 antibodies, with 165 distinct sequences, bound to SARS-CoV-2 RBD (Extended Data Fig. 3). These antibodies exhibited a wide range of RBD-binding activities. For example, many antibodies from P2B, P2C, P4A, P4B, P5A, P16A and P22A resulted in $A_{450}$ values well over 4.0, whereas no antibodies from P1A resulted in $A_{450}$ values over 4.0. There were insufficient antibodies from P3A and P8A for meaningful evaluation (Extended Data Fig. 3). Furthermore, there were substantial differences in expression of heavy-chain and light-chain genes across subjects. Some antibodies were clonally expanded and others remained relatively rare.
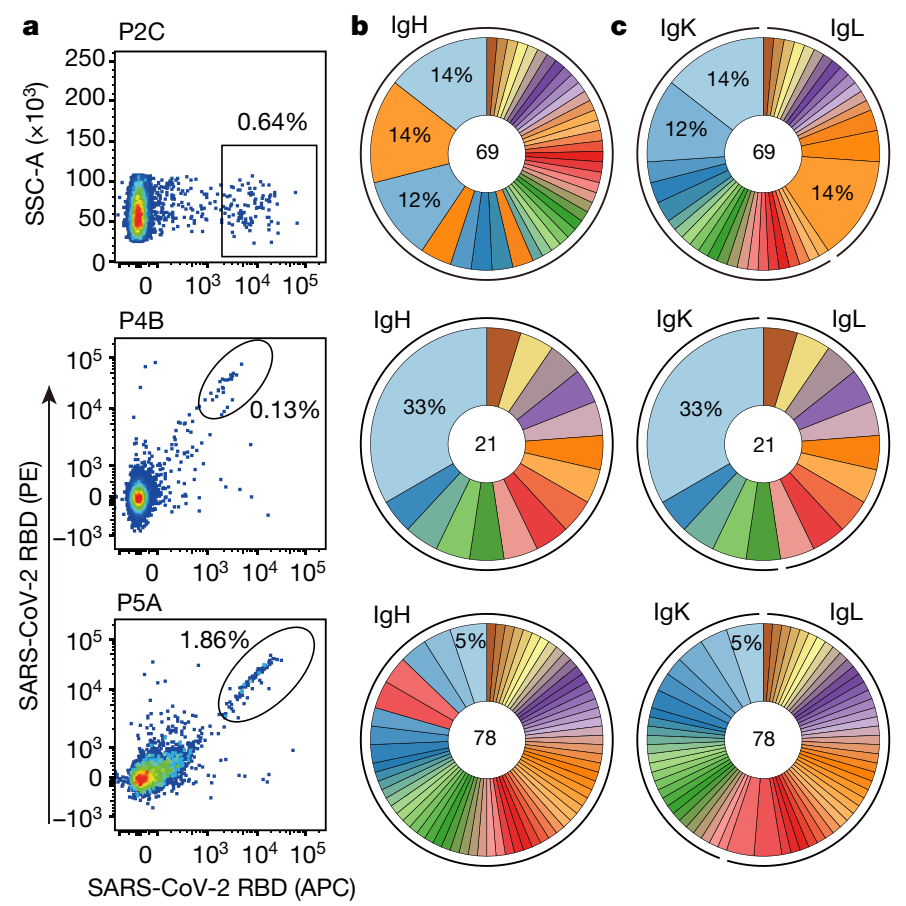

Fig. 2 |Single B cell antibody cloning and repertoire analysis. a, Isolated memory B cells from representative samples are outlined. One (top) or two (middle, bottom) fluorescently labelled RBD probes were used.SSC-A, side-scatter area. b, c, Repertoire analysis of antibody heavy (b) and light (c) chains. At the centre of each chart is the number of RBD-specific antibodies. Each slice represents a distinct clone and is proportional to the clone size. The percentages shown indicate the proportion of the expanded clone in relation to all isolated antibodies.

The expanded clones constituted varying fractions of the antibody repertoire among different patients (Fig. 2b, c). However, no single antibody family or group of antibody families stood out across patients, suggesting that individual patients had immunologically distinct responses.

We conducted more detailed characterization of samples from $\mathrm{P} 2$, as this individual had a large number of RBD-binding antibodies and was the only subject with three sequential samples. Of the 69 P2 antibodies, the majority (59\%) were scattered across branches and the remainder (41\%) were clonally expanded into three major clusters (Extended Data Fig. 4). The antibodies from the three time points (A, B and C) do not group together but rather interdigitate, suggesting that they are highly related. Three clones were significantly enriched (Extended Data Fig. 4), and each constituted 12-14\% of the tested repertoire (Fig. 2b, c). Their heavy-chain variable regions belong to the $\mathrm{V}_{\mathrm{H}} 1-2^{*} 06, \mathrm{~V}_{\mathrm{H}} 3-48^{*} 02$ and $\mathrm{V}_{\mathrm{H}} 3-9^{*} 01$ families. The corresponding kappa light chains (Igk) belong to $2-40^{*} 01,2 \mathrm{D}-40^{*} 01$ and $3-20^{*} 01$ families, and the lambda light chains (Igl) belong to $2-14 * 02$ families, with the respective joining segment kappa $4\left(\mathrm{~J}_{\mathrm{k}} 4\right)$, $\mathrm{J}_{\mathrm{k}} 5$ and joining segment lambda $1\left(\mathrm{~J}_{1} 1\right)$ (Extended Data Fig. 4$)$. These antibodies were identified in all three samples, indicating strong selection. Notably, somatic hypermutation (SHM) for $\mathrm{V}_{\mathrm{H}} 1-2^{*} 06$ was $0 \%$ and this cluster persisted during the study period. However, SHM was as high as $9.6 \%$ for $\mathrm{V}_{\mathrm{H}} 3-48^{*} 02$ and $3.8 \%$ for $\mathrm{V}_{\mathrm{H}} 3-9^{*} 01$, compared with the average of $2.2 \% \pm 3.3 \%$ among the $69 \mathrm{~V}_{\mathrm{H}}$ sequences. Lengths of complementarity-determining region 3 (CDR3) for $\mathrm{V}_{\mathrm{H}} 1-2^{*} 06$, $\mathrm{V}_{\mathrm{H}} 3-48^{*} 02$ and $\mathrm{V}_{\mathrm{H}} 3-9^{*} 01$ were 19 amino acid residues $(\mathrm{aa}), 16$ aa and 23 aa, respectively, compared with an average of $16 \pm 4$ aa (Extended Data Table 1). The longest CDR3 from the $\mathrm{V}_{\mathrm{H}} 3-9^{*} 01$ cluster was rich in tyrosine, suggesting that it forms hydrogen-bonding and hydrophobic interactions with the surrounding residues. 


\section{Binding and neutralizing properties}

We selected 13 of the $69 \mathrm{P} 2$ antibody sequences on the basis of their phylogenetic representation and distribution (Extended Data Fig. 4, asterisks). Five P1A antibody clones were used as controls. Dissociation constants with the SARS-CoV-2 RBD were obtained by surface plasmon resonance (SPR) (Extended Data Table 1). For P2 clones, the dissociation constant $\left(K_{\mathrm{d}}\right)$ ranged from 1.38 to $21.29 \mathrm{nM} . K_{\mathrm{d}}$ of P1 clones ranged from 8.48 to $260.50 \mathrm{nM}$ or was undetectable. Some germline clones with $0 \%$ divergence in both heavy and light chain genes (P2A-1A10, P2B-2G4, P2C-1A3 and P2C-1E1) had $K_{\mathrm{d}}$ values ranging from 2.47 to $21.29 \mathrm{nM}$, a similar range to the $K_{\mathrm{d}}$ values (1.38 to $17.57 \mathrm{nM}$ ) of clones with greater SHM (Extended Data Table 1). The $K_{\mathrm{d}}$ of representative clones (P2A-1A8, P2A-1A10 and P2A-1B3) from the 3 expanded clusters ranged from 4.65 to $8.91 \mathrm{nM}$, suggesting that expansion might have been driven by affinity maturation. We next measured antibody competition with ACE2, which was quantified as reduction in ACE2 and RBD binding (Extended Data Fig. 6, Extended Data Table 1). The most competitive antibodies included P2C-1F11, P2B-2F6, P2C-1A3 and P2A-1A10. Two of the three representative antibodies from the clonal expansion (P2A-1A10 and P2A-1B3) also competed strongly. The third (P2A-1A8) was a modest competitor. Many antibodies with low $K_{\mathrm{d}}$ values minimally competed with ACE2, suggesting that binding affinity does not predict ACE2-competing capacity. P1 controls demonstrated even lower competition with ACE2. No antibodies bound SARS-CoV or MERS-CoV RBDs, except P1A-1C7 $\left(K_{\mathrm{d}}=4.85 \mu \mathrm{M}\right)$, which had limited cross-reactivity with SARS-CoV RBD (Extended Data Fig. 5).

Consistent with findings from competition assays, antibody neutralization of pseudoviruses bearing SARS-CoV-2 spike protein varied considerably. Half-maximal inhibitory concentration $\left(\mathrm{IC}_{50}\right)$ values ranged from 0.03 to more than $50 \mu \mathrm{g} \mathrm{ml}^{-1}$ (Fig. 3a). P2C-1F11, P2B-2F6 and $\mathrm{P} 2 \mathrm{C}-1 \mathrm{~A} 3$ were the most potent, with $\mathrm{IC}_{50}$ values of $0.03,0.05$ and $0.62 \mu \mathrm{g} \mathrm{ml}^{-1}$, respectively. Overall, ACE2 competition correlated with neutralizing activity, although it did not do so exactly in all instances. Notably, no cross-neutralization was detected using pseudoviruses bearing SARS-CoV or MERS-CoV spike proteins (data not shown) or with cell-surface staining of trimeric SARS-CoV and MERS-CoV spike proteins (Supplementary Fig. 1).

We selected the top seven pseudovirus-neutralizing antibodies to measure inhibition of live SARS-CoV-2 using focus-reduction neutralization tests (FRNT) (Fig. 3b, Extended Data Fig. 7). Consistent with the respective pseudovirus assay data, $\mathrm{P} 2 \mathrm{C}-1 \mathrm{~F} 11, \mathrm{P} 2 \mathrm{~B}-2 \mathrm{~F} 6$ and P2C-1A3 were the most potent inhibitors of live SARS-CoV-2, with IC values $0.03,0.41$ and $0.28 \mu \mathrm{g} \mathrm{ml}^{-1}$, respectively (Fig. 3b, Extended Data Table 1). The remaining antibodies had moderate $\mathrm{IC}_{50}$ values ranging from 1.64 to $35.87 \mathrm{gg} \mathrm{ml}^{-1}$ (Fig. 3b, Extended Data Table 1). Finally, we determined whether these antibodies compete for similar epitopes by analysing them in a pairwise fashion using SPR. Competition was variable among the pairs (Extended Data Fig. 8). For example, P2C-1A3 was the most competitive, whereas $\mathrm{P} 2 \mathrm{C}-1 \mathrm{~F} 11$ was less competitive and only minimally competitive with $\mathrm{P} 2 \mathrm{C}-1 \mathrm{C} 10$. $\mathrm{P} 2 \mathrm{~B}-2 \mathrm{~F} 6$, another potent neutralizer, was broadly competitive with all antibodies tested. These results indicate recognition of both overlapping and distinct epitopes by these antibodies.

\section{Structural basis for neutralization}

We attempted to characterize the crystal structures of P2C-1F11, P2B-2F6 and P2C-1A3. However, we obtained crystals only with the complex of P2B-2F6 Fab and SARS-CoV-2 RBD. This structure was determined at $2.85 \AA$ resolution (Fig. 4a). P2B-2F6 primarily uses the heavy chain for RBD interactions. The paratope consists of 14 heavy chain residues (Y27, S28, S30, S31 and Y33 of heavy chain CDR 1 (HCDR1); H54 of HCDR2; and G102, I103, V105, V106 and P107 of HCDR3) and
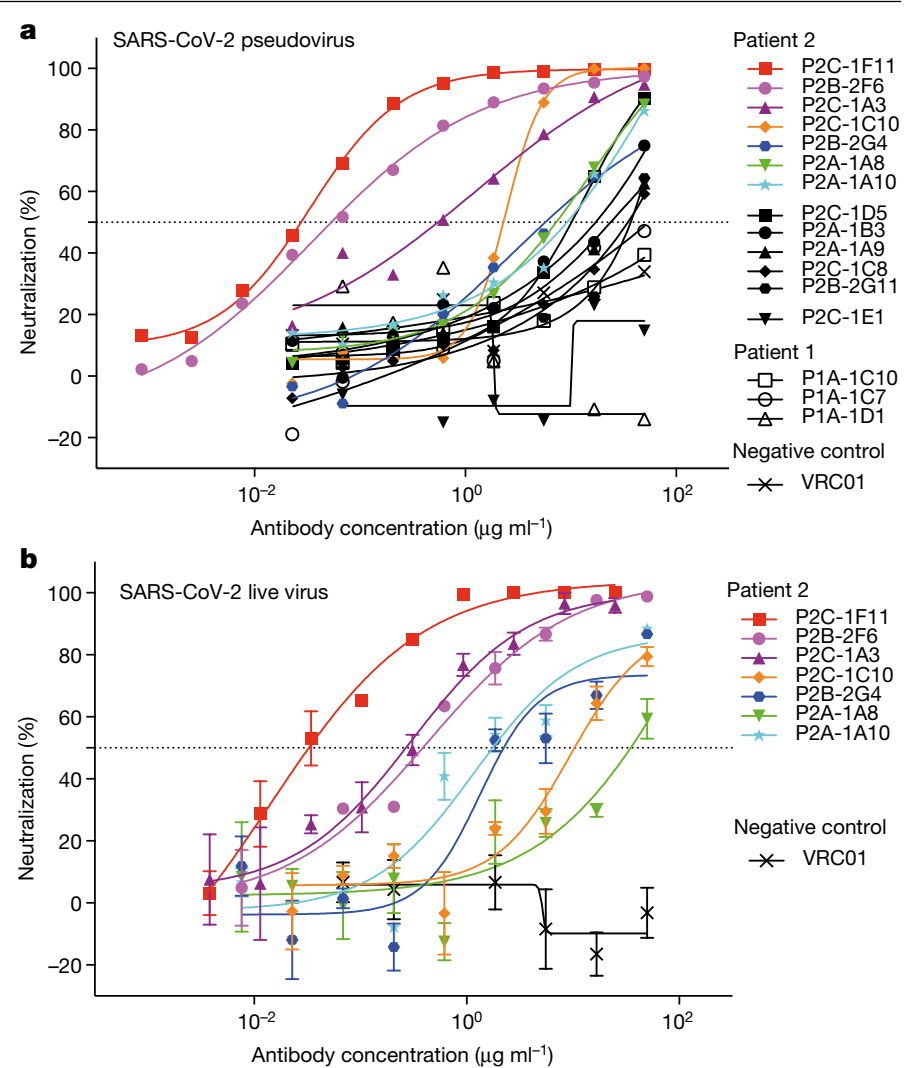

Fig. 3 | Antibody neutralization analysed with pseudovirus and live SARS-CoV-2.a, b, Neutralization activity against pseudovirus (a) or live SARS-CoV-2 (b). The dashed line indicates a 50\% reduction in viral infectivity. Results presented in a are representatives of two independent experiments. Data in $\mathbf{b}$ are mean \pm s.e.m. of $n=3$ experiments.

three light chain residues (G31, Y32 and N33 of light chain CDR 1). The buried RBD surface area is $626 \AA$. The epitope residues are all in the RBD receptor-binding motif, including residues $\mathrm{K} 444, \mathrm{G} 446, \mathrm{G} 447$, N448, Y449, N450, L452, V483, E484, G485, F490 and S494. P2B-2F6 attachment uses hydrophobic interactions around RBD residues $Y 449$, L452 and F490 and hydrophilic interactions at the interface (Fig. 4b, c, Supplementary Table 2). Superimposition of RBD-P2B-2F6 and RBDACE2 crystal structures indicated that $\mathrm{P} 2 \mathrm{~B}-2 \mathrm{~F} 6$ attachment clashes with ACE2 (Fig. 4d). The clash occurs between P2B-2F6 light chain (residues $\mathrm{R} 56, \mathrm{~S} 58, \mathrm{G} 59$, R63, S78 and G79) and ACE2 residues D67, K68, A71, K74, E110 and K114. The only overlapping residues recognized by P2B-2F6 and ACE2 are G446 and Y449, largely owing to angle differences as they approach RBD. However, high binding affinity between P2B-2F6 and RBD (5.14 nM), which is similar to that between ACE2 and RBD $(4.70 \mathrm{nM})$, probably precludes ACE2 receptor engagement. This is supported by the strong $\mathrm{P} 2 \mathrm{~B}-2 \mathrm{~F} 6$ competition with ACE2, as quantified by SPR (Extended Data Table1, Extended Data Fig. 6). We also superimposed the RBD and $\mathrm{P} 2 \mathrm{~B}-2 \mathrm{~F} 6$ crystal structure onto the cryo-electron microscopy structure of SARS-COV-2 spike (Protein Data Bank (PDB) ID: 6VSB), in which the RBD has 'up' and 'down' conformations. Unlike ACE2, which binds only the up conformation, the P2B-2F6 Fab binds both up and down conformations without clashing with the other spike monomers (Fig. 4e). These structural findings indicate that P2B-2F6 directly competes with spike for the ACE2 receptor.

\section{Discussion}

We characterized antibody responses in 8 patients infected with SARS-CoV-2 and isolated 206 monoclonal antibodies specific to 


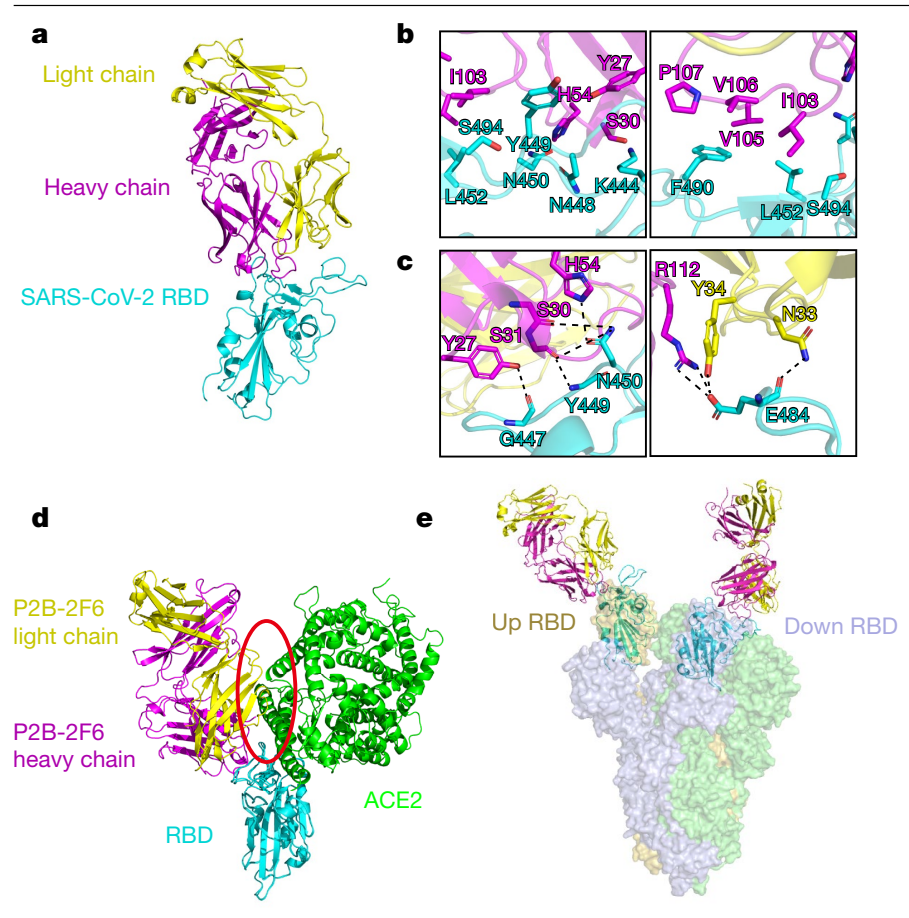

Fig. 4 | Crystal structure of P2B-2F6 in complex with SARS-CoV-2 RBD. a, Overall structure of $\mathrm{P} 2 \mathrm{~B}-2 \mathrm{~F} 6 \mathrm{Fab}$ in complex with SARS-CoV-2 RBD. b, Hydrophobic interactions around SARS-CoV-2 RBD residues Y449, L452 and F490 at the binding interface. $c$, Hydrogen bonds and salt bridges at the binding interface. d, Structural superposition of the RBD-P2B-2F6 and RBDACE2 crystal structures (PDB ID: 6MOJ) shows that clashes between ACE2 and P2B-2F6 would occur at the site within the red circle.e, The SARS-CoV-2 spike (PDB ID: 6VSB) is shown as a molecular surface, with protomers coloured light orange, blue or green. The RBD-P2B-2F6 complex could be aligned with both the up (light orange) and down (light blue) RBD in spike. The P2B-2F6 heavy chain is coloured magenta, $\mathrm{P} 2 \mathrm{~B}-2 \mathrm{~F} 6$ light chain is yellow, SARS-CoV-2 RBD is cyan and ACE2 is green.

the SARS-CoV-2 RBD. Bioinformatic and biologic characterization indicates that these antibodies are derived from diverse heavy- and light-chain families. Each patient appears to have unique antibody repertoire distribution patterns and there is no overall preference for a particular antibody family. Some clones appear only once, whereas others have undergone substantial clonal expansion. Over the course of infection, some clones remained virtually identical to their germline ancestors, while others diverged. CDR3 length also varied among clones. These genetic-level differences correlated with the respective binding and neutralizing activities. Antigen-binding affinities ranged from 1.38 to $21.29 \mathrm{nM}$, comparable with those of antibodies identified during acute infections ${ }^{16,17}$ but lower than those of antibodies resulting from chronic HIV-1 infection ${ }^{18-20}$. In general, competition with ACE2 is a better predictor than binding affinity for antibody virus-neutralizing potency. The most potent neutralizing antibodies (P2C-1F11, P2B-2F6 and P2C-1A3) were the most competitive with ACE2, indicating that blocking the interaction between RBD and ACE2 is a useful surrogate for neutralization. Analysis of the crystal structure of the complex of P2B-2F6 Fab and SARS-CoV-2 RBD supported competition with ACE2 binding. The structural clash between P2B-2F6 and ACE2 is expected to preclude RBD engagement with the ACE2 receptor. Substantial variations in competition for similar RBD epitopes or regions were also found between antibodies. The most potent antibody, $\mathrm{P} 2 \mathrm{C}-1 \mathrm{~F} 11$, did not target the same epitope as the relatively moderate antibody $\mathrm{P} 2 \mathrm{C}-1 \mathrm{C} 10$. These monoclonal antibodies could be combined for synergistic antiviral effect. As we screen more antibodies from study samples, we expect to identify more potent and diverse antibodies that could be developed for clinical interventions against SARS-CoV-2.

Most surprising was the absence of antibody cross-reactivity with RBDs from SARS-CoV and MERS-CoV. We expected some degree of cross-binding and even cross-neutralization between the two viruses, considering the sequential and structural similarities between SARS-CoV-2 and SARS-CoV RBDs. However, viral species-specific responses identified in patients suggest that these RBDs are immunologically distinct. If so, interventions must target each viral species differently to achieve maximum efficacy. These findings demonstrate why, to some extent, many previously isolated SARS-CoV antibodies fail to cross-neutralize SARS-CoV-2 despite detectable binding with the SARS-CoV-2 spike protein ${ }^{21}$. Conversely, we found that P2B-2F6 failed to cross-react with SARS-CoV RBD. Only 4 of the 12 epitope residues recognized by $\mathrm{P} 2 \mathrm{~B}-2 \mathrm{~F} 6$ are conserved, which probably explains the lack of cross-reactivity of P2B-2F6 with SARS-CoV. Cross-recognition between RBDs was also absent in plasma assays, further highlighting the immunological distinctiveness of RBDs from the three viruses. However, substantial plasma cross-reactivity was found with SARS-CoV trimeric spike protein and, to a lower degree, MERS-CoV trimeric spike proteins. This indicates that cross-reactivity probably occurs outside of RBD regions. Determining whether this cross-reactivity has protective implications requires further investigation.

Recent structural studies have examined the epitopes to which the human antibody CR3022 binds to the SARS-CoV-2 RBD ${ }^{22}$. The antibody was isolated from a patient infected with SARS-CoV, and demonstrated cross-binding to RBD but failed to neutralize SARS-CoV-2. To our knowledge, P2B-2F6 is the first reported antibody from a patient infected with SARS-CoV-2 whose epitope and interference with the ACE2 receptor have been characterized at the atomic level. We did not obtain crystal structures of two other potent neutralizing antibodies (P2C-1F11 and P2C-1A3); however, given their strong competition with $\mathrm{P} 2 \mathrm{~B}-2 \mathrm{~F} 6$, it is reasonable to hypothesize that they also act through interference in the interactions with RBD and ACE2. Finally, plasma samples from three patients with severe cases of COVID-19 (P1, P2 and P5) demonstrate increased binding to SARS-CoV-2 compared with plasma from patients with mild symptoms. However, we were unable to demonstrate a correlation between antibody response and disease progression. A larger cohort must be studied to identify the drivers and effects of any such association. This study will provide crucial reference points for antibody-based interventions against SARS-CoV-2 infection.

\section{Online content}

Any methods, additional references, Nature Research reporting summaries, source data, extended data, supplementary information, acknowledgements, peer review information; details of author contributions and competing interests; and statements of data and code availability are available at https://doi.org/10.1038/s41586-020-2380-z.

1. Li, Q. et al. Early transmission dynamics in Wuhan, China, of novel coronavirus-infected pneumonia. N. Engl. J. Med. 382, 1199-1207 (2020).

2. Zhou, P. et al. A pneumonia outbreak associated with a new coronavirus of probable bat origin. Nature 579, 270-273 (2020).

3. Guan, W.-J. et al. Clinical characteristics of coronavirus disease 2019 in China. N. Engl. J. Med. 382, 1708-1720 (2020).

4. Lu, R. et al. Genomic characterisation and epidemiology of 2019 novel coronavirus: implications for virus origins and receptor binding. Lancet 395, 565-574 (2020).

5. Hoffmann, M. SARS-CoV-2 cell entry depends on ACE2 and TMPRSS2 and is blocked by a clinically proven protease inhibitor. Cell 181, 271-280 (2020).

6. Walls, A. C. et al. Structure, function, and antigenicity of the SARS-CoV-2 Spike glycoprotein. Cell 181, 281-292 (2020).

7. Lan, J. et al. Structure of the SARS-CoV-2 spike receptor-binding domain bound to the ACE2 receptor. Nature 581, 215-220 (2020).

8. Wrapp, D. et al. Cryo-EM structure of the 2019-nCoV spike in the prefusion conformation Science 367, 1260-1263 (2020). 
9. Kirchdoerfer, R. N. et al. Stabilized coronavirus spikes are resistant to conformational changes induced by receptor recognition or proteolysis. Sci. Rep. 8, 15701 (2018).

10. Song, W., Gui, M., Wang, X. \& Xiang, Y. Cryo-EM structure of the SARS coronavirus spike glycoprotein in complex with its host cell receptor ACE2. PLoS Pathog. 14, e1007236 (2018).

11. Du, L. et al. The spike protein of SARS-CoV-a target for vaccine and therapeutic development. Nat. Rev. Microbiol. 7, 226-236 (2009).

12. $\mathrm{Xu}$, J. et al. Antibodies and vaccines against Middle East respiratory syndrome coronavirus. Emerg. Microbes Infect. 8, 841-856 (2019).

13. Chan, J. F.-W. et al. A familial cluster of pneumonia associated with the 2019 novel coronavirus indicating person-to-person transmission: a study of a family cluster. Lancet 395, 514-523 (2020)

14. Sanders, R. W. et al. Stabilization of the soluble, cleaved, trimeric form of the envelope glycoprotein complex of human immunodeficiency virus type 1. J. Virol. 76, 8875-8889 (2002).

15. Kong, L. et al. Key gp120 Glycans pose roadblocks to the rapid development of VRC01-class antibodies in an HIV-1-infected Chinese donor. Immunity 44, 939-950 (2016).

16. Yu, L. et al. Delineating antibody recognition against Zika virus during natural infection. JCl Insight 2, e93042 (2017).
17. Stettler, K. et al. Specificity, cross-reactivity, and function of antibodies elicited by Zika virus infection. Science 353, 823-826 (2016).

18. Scheid, J. F. et al. Broad diversity of neutralizing antibodies isolated from memory B cells in HIV-infected individuals. Nature 458, 636-640 (2009).

19. Wu, X. et al. Focused evolution of HIV-1 neutralizing antibodies revealed by structures and deep sequencing. Science 333, 1593-1602 (2011).

20. Liao, H.-X. et al. Co-evolution of a broadly neutralizing HIV-1 antibody and founder virus. Nature 496, 469-476 (2013).

21. Tian, X. et al. Potent binding of 2019 novel coronavirus spike protein by a SARS coronavirus-specific human monoclonal antibody. Emerg. Microbes Infect. 9, 382-385 (2020).

22. Yuan, M. et al. A highly conserved cryptic epitope in the receptor binding domains of SARS-CoV-2 and SARS-CoV. Science 368, 630-633 (2020).

Publisher's note Springer Nature remains neutral with regard to jurisdictional claims in published maps and institutional affiliations.

(C) The Author(s), under exclusive licence to Springer Nature Limited 2020 


\section{Methods}

\section{Data reporting}

No statistical methods were used to predetermine sample size. The experiments were not randomized. The investigators were not blinded to allocation during experiments and outcome assessment.

\section{Study approval}

This study received approval from the Research Ethics Committee of Shenzhen Third People's Hospital, China (approval number: 2020084). The research was conducted in strict accordance with the rules and regulations of the Chinse government for the protection of human subjects. The study subjects agreed and signed the written informed consents for research use of their blood samples.

\section{Patients and blood samples}

The study enrolled a total of eight patients aged 10 to 66 years old infected with SARS-CoV-2 in January 2020 (Supplementary Table 1). A plasma sample from a healthy control individual was also included. Of these eight patients, six (P1-P4, P8 and P16) had Wuhan exposure history through personal visits and two had direct contact with individuals from Wuhan. Four subjects (P1-P4) were part of a family cluster (P1-P5) infected while visiting Wuhan, and subsequently transmitted the infection to P5 after returning to Shenzhen ${ }^{13}$. All patients were hospitalized at Shenzhen Third People's Hospital, the designated city hospital for the treatment of patients with COVID-19, three to nine days after symptom onset. All patients presented with fever, fatigue and dry cough, and three (P1,P2 and P5) developed severe pneumonia. Four patients (P1,P2, P5 and $\mathrm{P} 22)$ were 60 years of age or older, of whom three (P1, P2 and P22) had underlying disease such as hypertension. SARS-CoV-2 infection status was verified by RT-PCR of nasopharyngeal swab and throat swab specimens. No patient had detectable influenza A, B, respiratory syncytial virus (RSV), or adenovirus co-infections. Chest computed tomographic scans showed varying degrees of bilateral lung patchy shadows or opacity. All patients received interferon and ribavirin and/or methylprednisolone treatments, recovered and were discharged except for $\mathrm{P} 1$, who succumbed to disease in hospital. Single (P1, P3, P5, P8, P16 and P22) or sequential ( $\mathrm{P} 2$ and $\mathrm{P} 4$ ) blood samples were collected during hospitalization and follow-up visits and separated into plasma and peripheral blood mononuclear cells (PBMCs) by Ficoll-Hypaque gradient (GE Healthcare) centrifugation. All plasma samples were heat-inactivated at $56^{\circ} \mathrm{C}$ for $1 \mathrm{~h}$ before being stored at $-80^{\circ} \mathrm{C}$. PBMCs were maintained in freezing medium and stored in liquid nitrogen until use.

\section{Recombinant RBDs and trimeric spike from SARS-CoV-2, SARS-CoV, and MERS-CoV and receptor ACE2}

Recombinant RBDs and trimeric spike for MERS-CoV, SARS-CoV, and SARS-CoV-2 and the N-terminal peptidase domain of human ACE2 (residues Ser19-Asp615) were expressed using the Bac-to-Bac baculovirus system (Invitrogen) as previously described ${ }^{23,24}$. SARS-CoV-2 RBD (residues Arg319-Phe541) containing the gp67 secretion signal peptide and a C-terminal hexahistidine or strep tag was inserted into pFastBac-Dual vectors (Invitrogen) and transformed into DH1OBac component cells. The bacmid was extracted and further transfected into Sf 9 cells using Cellfectin II Reagents (Invitrogen). The recombinant viruses were collected from the transfected supernatant and amplified to generate high-titre virus stock. Viruses were then used to infect High Five cells for RBD and trimeric spike expression. Secreted RBD and trimeric spike were collected from the supernatant and purified by gel filtration chromatography as previously reported ${ }^{24,25}$.

ELISA analysis of plasma and antibody binding to RBD, trimeric spike and NP proteins

The recombinant RBDs and trimeric spike derived from SARS-CoV-2, SARS-CoV and MERS-CoV and the SARS-CoV-2 nucleoprotein
(Sino Biological) were diluted to final concentrations of $0.5 \mu \mathrm{g} \mathrm{ml}^{-1}$ or $2 \mu \mathrm{g} \mathrm{ml}^{-1}$, then coated onto 96 -well plates and incubated at $4{ }^{\circ} \mathrm{C}$ overnight. Samples were washed with PBS-T (PBS containing 0.05\% Tween-20) and blocked with blocking buffer (PBS containing 5\% skim milk and 2\% BSA) at room temperature for $1 \mathrm{~h}$. Either serially diluted plasma samples or isolated monoclonal antibodies were added the plates and incubated at $37^{\circ} \mathrm{C}$ for $1 \mathrm{~h}$. Wells were then incubated with secondary anti-human IgG labelled with HRP (ZSGB-BIO) and TMB substrate (Kinghawk) and optical density (OD) was measured by a spectrophotometer at $450 \mathrm{~nm}$ and $630 \mathrm{~nm}$. The serially diluted plasma from healthy individuals or previously published monoclonal antibodies against SARS-CoV (S230), MERS-CoV (Mab-GD33) and HIV-1 (VRC01) were used as controls.

\section{Isolation of RBD-specific single B cells by FACS}

RBD-specific single B cells were sorted as previously described ${ }^{15}$. In brief, PBMCs from infected individuals were collected and incubated with an antibody and RBD cocktail for identification of RBD-specific $B$ cells. The cocktail consisted of CD19-PE-Cy7, CD3-Pacific Blue, CD8-Pacific Blue, CD14-Pacific Blue, CD27-APC-H7, IgG-FITC (BD Biosciences) and the recombinant RBD-Strep or RBD-His described above. Three consecutive staining steps were conducted. The first was a LIVE/DEAD Fixable Dead Cell Stain Kit (Invitrogen) in $50 \mu$ l phosphate-buffered saline (PBS) applied at room temperature for 20 min to exclude dead cells. The second used an antibody and RBD cocktail for an additional $30 \mathrm{~min}$ at $4{ }^{\circ} \mathrm{C}$. The third staining at $4{ }^{\circ} \mathrm{C}$ for $30 \mathrm{~min}$ involved either: streptavidin-APC (eBioscience) and/or streptavidin-PE (BD Biosciences) to target the Strep tag of RBD, or anti-His-APC and anti-His-PE antibodies (Abcam) to target the His tag of RBD. The stained cells were washed and resuspended in PBS before being strained through a $70-\mu \mathrm{m}$ cell mesh (BD Biosciences). RBD-specific single $B$ cells were gated as $\mathrm{CD}{ }^{+} \mathrm{CD}^{-} \mathrm{CD}^{-} \mathrm{CD}^{-} 4^{-} \mathrm{IgG}^{+} \mathrm{RBD}^{+}$and sorted into 96 -well PCR plates containing $20 \mu \mathrm{l}$ of lysis buffer $(5 \mu \mathrm{l}$ of $5 \times$ first strand buffer, $0.5 \mu \mathrm{l}$ of RNase out, $1.25 \mu \mathrm{l}$ of $0.1 \mathrm{M} \mathrm{DTT}$ (Invitrogen) per well and $0.0625 \mu \mathrm{l}$ of Igepal (Sigma). Plates were then snap-frozen on dry ice and stored at $-80^{\circ} \mathrm{C}$ until the reverse transcription reaction.

\section{Single B cell PCR, cloning and expression of monoclonal antibodies}

The IgG heavy and light chain variable genes were amplified by nested PCR and cloned into linear expression cassettes or expression vectors to produce full IgG1 antibodies as previously described ${ }^{15}$.Specifically, all second round PCR primers containing tag sequences were used to produce the linear Ig expression cassettes by overlapping PCR. Separate primer pairs containing the specific restriction enzyme cutting sites (heavy chain, $5^{\prime}$-Agel/3'-SalI; kappa chain, 5'-Agel/3'-BsiWI; and lambda chain, $5^{\prime}$-Agel/ $/ 3^{\prime}$-Xhol) were used to amplify the cloned PCR products. The PCR products were purified and cloned into the backbone of antibody expression vectors containing the constant regions of human IgG1. Overlapping PCR products of paired heavy and light chain expression cassettes were co-transfected into 293T cells (ATCC) grown in 24-well plates. Antigen-specific ELISA was used to detect the binding capacity of transfected culture supernatants to SARS-CoV-2 RBD. Monoclonal antibodies were produced by transient transfection of $293 \mathrm{~F}$ cells (Life Technologies) with equal amounts of paired heavy and light chain plasmids. Antibodies in the culture supernatant was purified by affinity chromatography using Protein A beads columns (National Engineering Research Center for Biotechnology) according to the manufacturer's protocol. Concentrations were determined by BCA Protein Assay Kits (Thermo Scientific). SARS-CoV, MERS-CoV, and HIV-1 monoclonal antibodies were also included as controls. SARS-CoV antibodies (S230 and m396) previously isolated by others ${ }^{26}$ were synthesized, expressed in 293T cells and purified by protein A chromatography. MERS-CoV antibodies (Mab-GD33) were derived from previous reports ${ }^{27}$. HIV-1 antibody VRC01 was a broadly neutralizing antibody 
directly isolated from a patient targeting the CD4 binding site of envelope glycoprotein ${ }^{28}$.

\section{Antibody binding kinetics, epitope mapping, and competition} with receptor ACE2 measured by SPR

The binding kinetics and affinity of monoclonal antibodies to SARS-CoV-2 RBD were analysed by SPR (Biacore T200, GE Healthcare). Specifically, purified RBDs were covalently immobilized to a CM5 sensor chip via amine groups in $10 \mathrm{mM}$ sodium acetate buffer ( $\mathrm{pH} 5.0)$ for a final RU around 250. SPR assays were run at a flow rate of $30 \mu \mathrm{l} / \mathrm{min}$ in HEPES buffer. The sensograms were fit in a 1:1 binding model with BIA Evaluation software (GE Healthcare). For epitope mapping, two different antibodies were sequentially injected and monitored for binding activity to determine whether the two monoclonal antibodies recognized separate or closely-situated epitopes. To determine competition with the human ACE2 peptidase domain, SARS-CoV-2 RBD was immobilized to a CM5 sensor chip via amine group for a final $\mathrm{RU}$ around 250. Antibodies $(1 \mu \mathrm{M})$ were injected onto the chip until binding steady-state was reached. ACE2 $(2 \mu \mathrm{M})$, which was produced and purified as above, was then injected for $60 \mathrm{~s}$. Blocking efficacy was determined by comparison of response units with and without prior antibody incubation.

\section{Analysis of plasma and antibody binding to cell surface expressed trimeric spike protein}

HEK 293T cells were transfected with expression plasmid encoding the full length spike of SARS-CoV-2, SARS-CoV or MERS-CoV and incubated at $37^{\circ} \mathrm{C}$ for $36 \mathrm{~h}$. The cells were removed from the plate using trypsin and distributed into 96-well plates for the individual staining. Cells were washed twice with $200 \mu$ l staining buffer (PBS with $2 \%$ heated-inactivated FBS) between each of the following. The cells were stained at room temperature for $30 \mathrm{~min}$ in $100 \mu \mathrm{l}$ staining buffer with 1:100 dilutions of plasma or $20 \mu \mathrm{g} / \mathrm{ml}$ monoclonal antibodies. The cells were then stained with PE labelled anti-human IgG Fc secondary antibody (Biolegend) at a 1:20 dilution in $50 \mu \mathrm{l}$ staining buffer at room temperature for $30 \mathrm{~min}$. Finally, the cells were re-suspended and analysed with FACS Calibur instrument (BD Biosciences) and FlowJo 10 software (FlowJo). HEK 293T cells without transfection were also stained as background control.S230 and m396 targeting the RBD of SARS-CoV Spike $^{26}$ and Mab-GD33 targeting the RBD of MERS-CoV Spike ${ }^{27}$ were used as positive primary antibody controls, while VRC01 targeting HIV-1 env $^{28}$ was used as an irrelevant primary antibody control.

\section{Neutralization activity of monoclonal antibodies against pseudovirus SARS-CoV-2}

SARS-CoV-2, SARS-CoV, and MERS-CoV pseudovirus were generated by co-transfection of human immunodeficiency virus backbones expressing firefly luciferase (pNL43R-E-luciferase) and pcDNA3.1 (Invitrogen) expression vectors encoding the respective spike proteins into 293 T cells (ATCC) ${ }^{24,29,30}$. Viral supernatants were collected $48 \mathrm{~h}$ later. Viral titers were measured as luciferase activity in relative light units (Bright-Glo Luciferase Assay Vector System, Promega Biosciences). Control envelope glycoproteins derived from human immunodeficiency virus (HIV)-1 and their corresponding pseudoviruses were produced in the same manner. Control monoclonal antibodies included VRC01 $(1 \mathrm{mg} / \mathrm{ml})$ against HIV-1 ${ }^{28} ; \mathrm{S} 230(0.15 \mu \mathrm{g} / \mathrm{ml})$ against SARS-CoV ${ }^{26}$; and Mers-GD33 $(12.35 \mu \mathrm{g} / \mathrm{ml})$ against MERS-CoV ${ }^{27}$. Neutralization assays were performed by incubating pseudoviruses with serial dilutions of purified monoclonal antibodies at $37^{\circ} \mathrm{C}$ for $1 \mathrm{~h}$. Huh7 cells for SARS-CoV-2, SARS-CoV, and MERS-CoV pseudovirus and Ghost cells for HIV-1 pseudovirus (ATCC) (approximately $1.5 \times 10^{4}$ per well) were added in duplicate to the virus-antibody mixture. Half-maximal inhibitory concentrations $\left(\mathrm{IC}_{50}\right.$ ) of the evaluated monoclonal antibodies were determined by luciferase activity $48 \mathrm{~h}$ after exposure to virus-antibody mixture using GraphPad Prism 7 (GraphPad Software).

\section{Neutralization activity of monoclonal antibodies against live} SARS-CoV-2

SARS-CoV-2 FRNT was performed in a certified Biosafety level 3 laboratory. Neutralization assays against live SARS-CoV-2 were conducted using a clinical isolate (Beta/Shenzhen/SZTH-003/2020, EPI_ISL_406594 at GISAID) previously obtained from a nasopharyngeal swab of an infected patient. Serial dilutions of testing antibodies were conducted, mixed with $75 \mu$ l of SARS-CoV- $2\left(8 \times 10^{3}\right.$ focus-forming units $(\mathrm{FFU}) / \mathrm{ml}$ ) in 96-well microwell plates and incubated for $1 \mathrm{~h}$ at $37^{\circ} \mathrm{C}$. Mixtures were then transferred to 96 -well plates seeded with Vero $\mathrm{E} 6$ cells and allowed absorption for $1 \mathrm{~h}$ at $37^{\circ} \mathrm{C}$. Inoculums were then removed before adding the overlay media ( $100 \mu$ I MEM containing $1.6 \%$ carboxymethylcellulose, $\mathrm{CMC}$ ). The plates were then incubated at $37^{\circ} \mathrm{C}$ for $24 \mathrm{~h}$. Cells were fixed with $4 \%$ paraformaldehyde solution for $30 \mathrm{~min}$, and overlays were removed. Cells were permeabilized with $0.2 \%$ Triton X-100 and incubated with cross-reactive rabbit anti-SARS-CoV-N IgG (Sino Biological) for $1 \mathrm{~h}$ at room temperature before adding HRP-conjugated goat anti-rabbit IgG $(\mathrm{H}+\mathrm{L})$ antibody (Jackson ImmunoResearch). Cells were further incubated at room temperature. The reactions were developed with KPL TrueBlue Peroxidase substrates (Seracare Life Sciences). The numbers of SARS-CoV-2 foci were calculated using an EliSpot reader (Cellular Technology).

\section{Gene family usage and phylogenetic analysis of monoclonal antibodies}

The program IMGT/V-QUEST (http://www.imgt.org/IMGT_vquest/ vquest) was used to analyse germline gene, germline divergence or degree of SHM, the framework region (FR) and the loop length of CDR3 for each antibody clone. The IgG heavy and light chain variable genes were aligned using Clustal $W$ in the BioEdit sequence analysis package (https://bioedit.software.informer.com/7.2/). Phylogenetic analyses were performed by the maximum likelihood method using MEGAX (molecular evolutionary genetics analysis across computing platforms). Several forms of the phylogenetic trees are presented for clarity.

\section{Antibody production}

The production of antibodies was conducted as previously described ${ }^{24}$. The genes encoding the heavy and light chains of isolated antibodies were separately cloned into expression vectors containing IgG1 constant regions and the vectors were transiently transfected into $293 \mathrm{~F}$ cells using polyethylenimine (PEI) (Sigma). After $72 \mathrm{~h}$, the antibodies secreted into the supernatant were collected and captured by protein A Sepharose (GE Healthcare). The bound antibodies were eluted and further purified by gel-filtration chromatography using a Superdex 200 High Performance column (GE Healthcare). The purified antibodies were either used in binding or neutralizing assays.

\section{Crystallization and data collection}

The SARS-CoV-2 RBD and the Fab fragment of P2B-2F6 were mixed at a molar ratio of $1: 1.2$, incubated for $2 \mathrm{~h}$ at $4{ }^{\circ} \mathrm{C}$ and further purified by gel-filtration chromatography. The purified complex concentrated to approximately $10 \mathrm{mg} / \mathrm{ml}$ in HBS buffer (10 mM HEPES, pH 7.2, $150 \mathrm{mM}$ $\mathrm{NaCl}$ ) was used for crystallization. The screening trials were performed at $18{ }^{\circ} \mathrm{C}$ using the sitting-drop vapour-diffusion method by mixing $0.2 \mu \mathrm{l}$ of protein with $0.2 \mu \mathrm{l}$ of reservoir solution. Crystals were successfully obtained in $0.2 \mathrm{M}$ magnesium formate dihydrate, $0.1 \mathrm{M}$ sodium acetate trihydrate, $\mathrm{pH}$ 4.0,18\% PEG5000mme. Crystals were collected, soaked briefly in mother liquid with $20 \%$ glycerol, and flash-frozen in liquid nitrogen. Diffraction data were collected at $100 \mathrm{~K}$ and at a wavelength of 1.071 Å on the BL17U1 beam line of the Shanghai Synchrotron Research Facility (SSRF). Diffraction data was auto-processed with aquarium pipeline ${ }^{31}$ and the data processing statistics are listed in Extended Data Table 2 . 


\section{Structure determination and refinement}

The structure was determined by the molecular replacement method with PHASER in CCP4 suite ${ }^{32}$. The search models were the SARS-CoV-2 RBD structure (PDB ID: 6MOJ) and the structures of the variable domain of the heavy and light chains available in the PDB with the highest sequence identities. Subsequent model building and refinement were performed using COOT and PHENIX, respectively ${ }^{33,34}$. Final Ramachandran statistics: $94.13 \%$ favoured, $5.87 \%$ allowed and $0.00 \%$ outliers for the final structure. The structural refinement statistics are listed in Extended Data Table 2. All structural figures were generated using PyMOL $^{35}$.

\section{Reporting summary}

Further information on research design is available in the Nature Research Reporting Summary linked to this paper.

\section{Data availability}

The coordinates and structure factor files for the complex of SARS-CoV-2 RBD with P2B-2F6 have been deposited in the Protein Data Bank under accession number 7BWJ. We are applying for patent protection for some of the antibodies. Reagents and information presented in this study are available upon reasonable request from the authors.

23. Wang, N. et al. Structure of MERS-CoV spike receptor-binding domain complexed with human receptor DPP4. Cell Res. 23, 986-993 (2013).

24. Jiang, L. et al. Potent neutralization of MERS-CoV by human neutralizing monoclonal antibodies to the viral spike glycoprotein. Sci Transl Med 6, 234ra59 (2014).

25. Gui, M. et al. Cryo-electron microscopy structures of the SARS-CoV spike glycoprotein reveal a prerequisite conformational state for receptor binding. Cell Res. 27, 119-129 (2017).

26. Zhu, Z. et al. Potent cross-reactive neutralization of SARS coronavirus isolates by human monoclonal antibodies. Proc. Natl Acad. Sci. USA 104, 12123-12128 (2007).

27. Niu, P. et al. Ultrapotent human neutralizing antibody repertoires against Middle East respiratory syndrome coronavirus from a recovered patient. J. Infect. Dis. 218, 1249-1260 (2018).

28. Wu, X. et al. Rational design of envelope identifies broadly neutralizing human monoclonal antibodies to HIV-1. Science 329, 856-861 (2010).

29. Jia, W. et al. Single intranasal immunization with chimpanzee adenovirus-based vaccine induces sustained and protective immunity against MERS-CoV infection. Emerg. Microbes Infect. 8, 760-772 (2019).
30. Zhang, L. et al. Antibody responses against SARS coronavirus are correlated with disease outcome of infected individuals. J. Med. Virol. 78, 1-8 (2006).

31. McCoy, A. J. et al. Phaser crystallographic software. J. Appl. Crystallogr. 40, 658-674 (2007).

32. Cohen, S. X. et al. ARP/WARP and molecular replacement: the next generation. Acta Crystallogr. D 64, 49-60 (2008)

33. Emsley, P. \& Cowtan, K. Coot: model-building tools for molecular graphics. Acta Crystallogr. D 60, 2126-2132 (2004).

34. Adams, P. D. et al. PHENIX: building new software for automated crystallographic structure determination. Acta Crystallogr. D 58, 1948-1954 (2002).

35. Janson, G., Zhang, C., Prado, M. G. \& Paiardini, A. PyMod 2.0: improvements in protein sequence-structure analysis and homology modeling within PyMOL. Bioinformatics $\mathbf{3 3}$, 444-446 (2017)

Acknowledgements We acknowledge the work and contribution of all the health providers from Shenzhen Third People's Hospital. We also thank patients for their active participation. This study was supported by Bill \& Melinda Gates Foundation, the Science and Technology Innovation Committee of Shenzhen Municipality (202002073000002), and by Tsinghua University Initiative Scientific Research Program (20201080053). This work is also partially supported by the National Natural Science Foundation Award (81530065, 91442127 and 81672037), Beijing Municipal Science and Technology Commission (171100000517-001 and 171100000517-003), Beijing Advanced Innovation Center for Structural Biology at Tsinghua University, the National Key Plan for Scientific Research and Development of China (grant number 2016YFD0500307), Tencent Foundation, Shuidi Foundation and TH Capital. The funders had no role in study design, data collection, data analysis, data interpretation or writing of the report.

Author contributions L.Z., Z.Z., X.W., L.L. and S.Z. conceived and designed the study. B.J., Q.Z. and J.G. performed most of the experiments, with assistance from R.W., J.S., X.G., Jiazhen Y., S. Shan, B.Z., S. Song, X.S. and X.T. J.S. and Jincun Z. performed and analysed the live SARS-CoV-2 neutralization assay. Jinfang Y., J.G., J.L. and X.W. provided assistance with production of RBD and trimeric spike protein. J.G. with assistance from R.W. solved and analysed the crystal structure of the antibody-RBD complex. Jinfang Y. and L.L. had critical roles in recruitment and clinical management of the study subjects. H.W. and Juanjuan Z. were in charge of sample collection and processing. Y.W. provided additional pseudovirus assays for measuring neutralizing activity against SARS-CoV-2. B.J., Q.Z., J.G., J.S., Jincun Z., X.W., Z.Z. and L.Z. had full access to data in the study, generated figures and tables, and take responsibility for the integrity and accuracy of the data presentation. X.W., L.Z. and Z.Z. wrote the manuscript. All authors reviewed and approved the final version of the manuscript.

Competing interests Patent applications have been filed that cover some of the antibodies presented here.

\section{Additional information}

Supplementary information is available for this paper at https://doi.org/10.1038/s41586-0202380-z.

Correspondence and requests for materials should be addressed to X.W., Z.Z. or L.Z. Peer review information Nature thanks Peter Colman and the other, anonymous, reviewer(s) for their contribution to the peer review of this work. Peer reviewer reports are available. Reprints and permissions information is available at http://www.nature.com/reprints. 

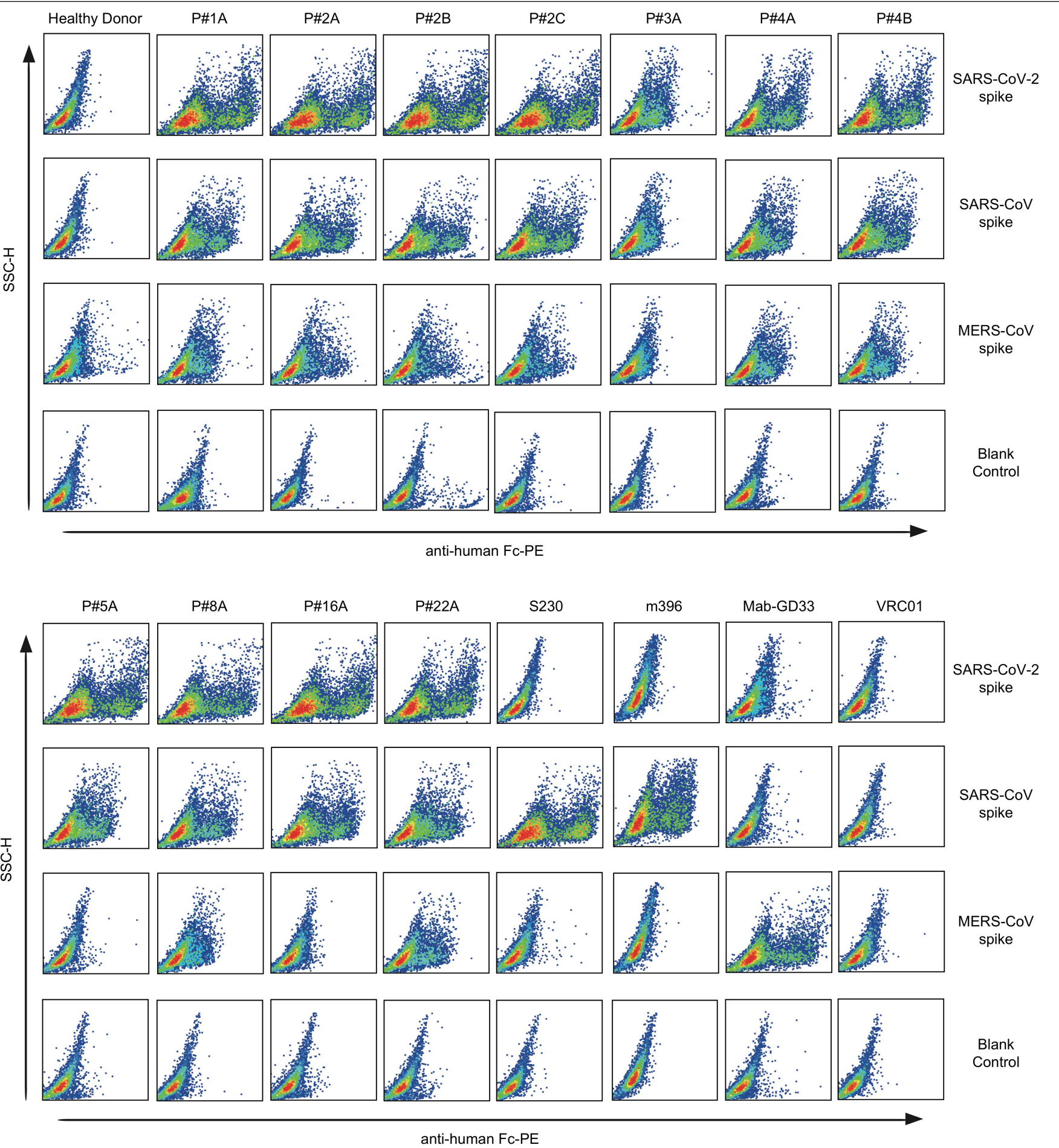

Extended Data Fig. 1 | Analysis of plasma binding to cell surface expressed trimeric spike protein. HEK 293T cells transfected with expression plasmid encoding the full length spike of SARS-CoV-2, SARS-CoV or MERS-CoV were incubated with 1:100 dilutions of plasma from the study subjects. The cells were then stained with PE labelled anti-human IgG Fc secondary antibody and analysed by FACS. Positive control antibodies include S230 and m396 targeting the RBD of SARS-CoV spike, and Mab-GD33 targeting the RBD of MERS-CoV spike. VRC01 is negative control antibody targeting HIV-1 envelope glycoprotein. Results presented are representatives of two independent experiments. 

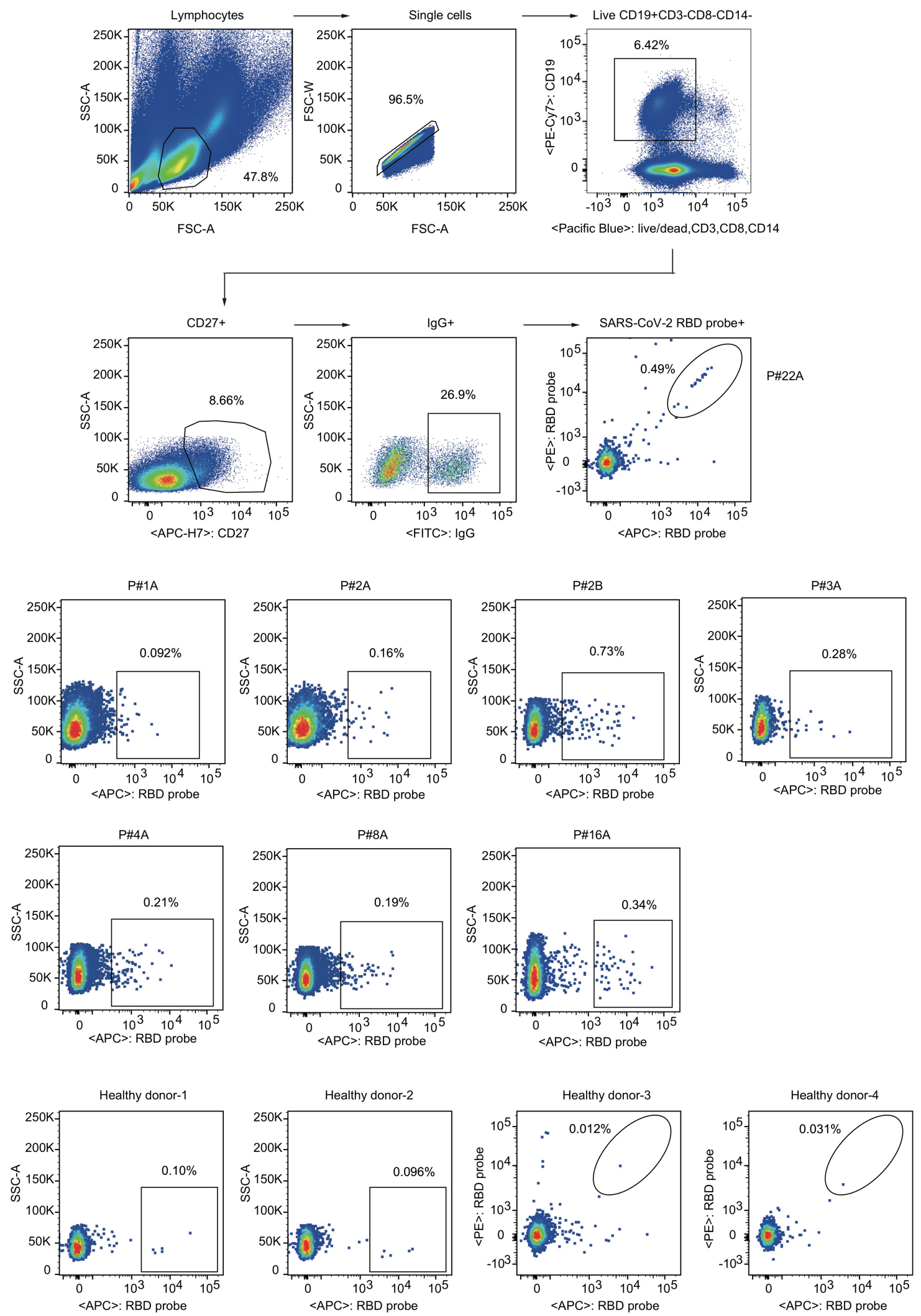

Extended Data Fig. 2 | Cell isolation. Gating strategy for isolation of RBD-specific memory B cells through FACS, calibrated with the background controls from healthy individuals. The isolated memory B cells from representative samples are highlighted in boxes or ovals depending on the RBD

probe used with one or two fluorophores. Samples were named as either A, B, or C depending on collection sequence. FSC-W: forward scatter width. SSC-A: side scatter area. 


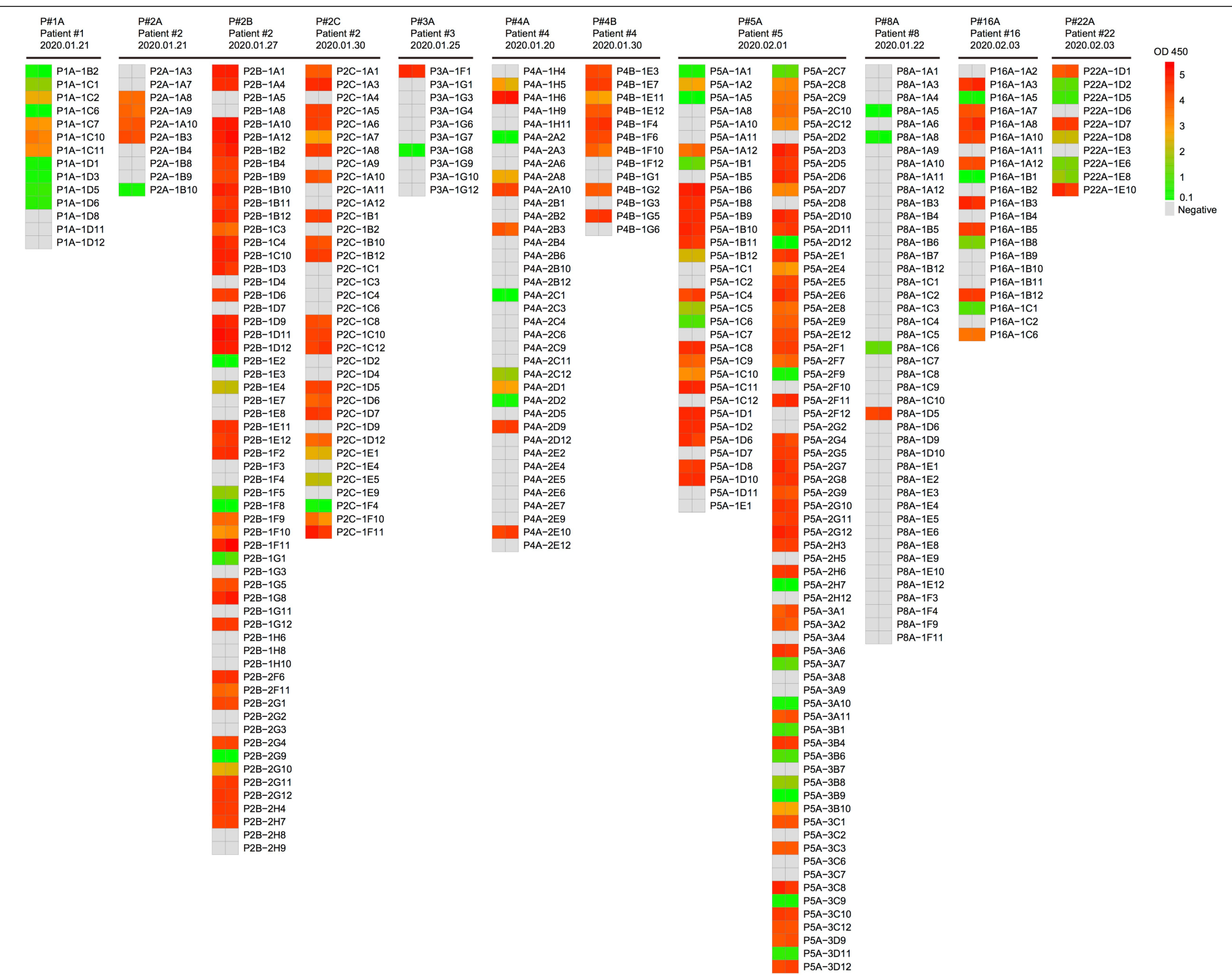

Extended Data Fig. 3 | ELISA screening of SARS-CoV-2 RBD-specific antibodies in the supernatant of transfected cells. The study subjects and the date of sampling are indicated on the top. Samples were named as either A, $\mathrm{B}$, or C depending on collection sequence. Antibodies tested for each sample are aligned in one vertical column whenever possible. For each evaluated antibody, at least two replicates were performed and are presented adjacently on the same row. Binding activities were assessed by OD 450 and indicated by the colour scheme on the right. Negatives (no binding activity) are shown in grey for OD 450 values less than 0.1 . 


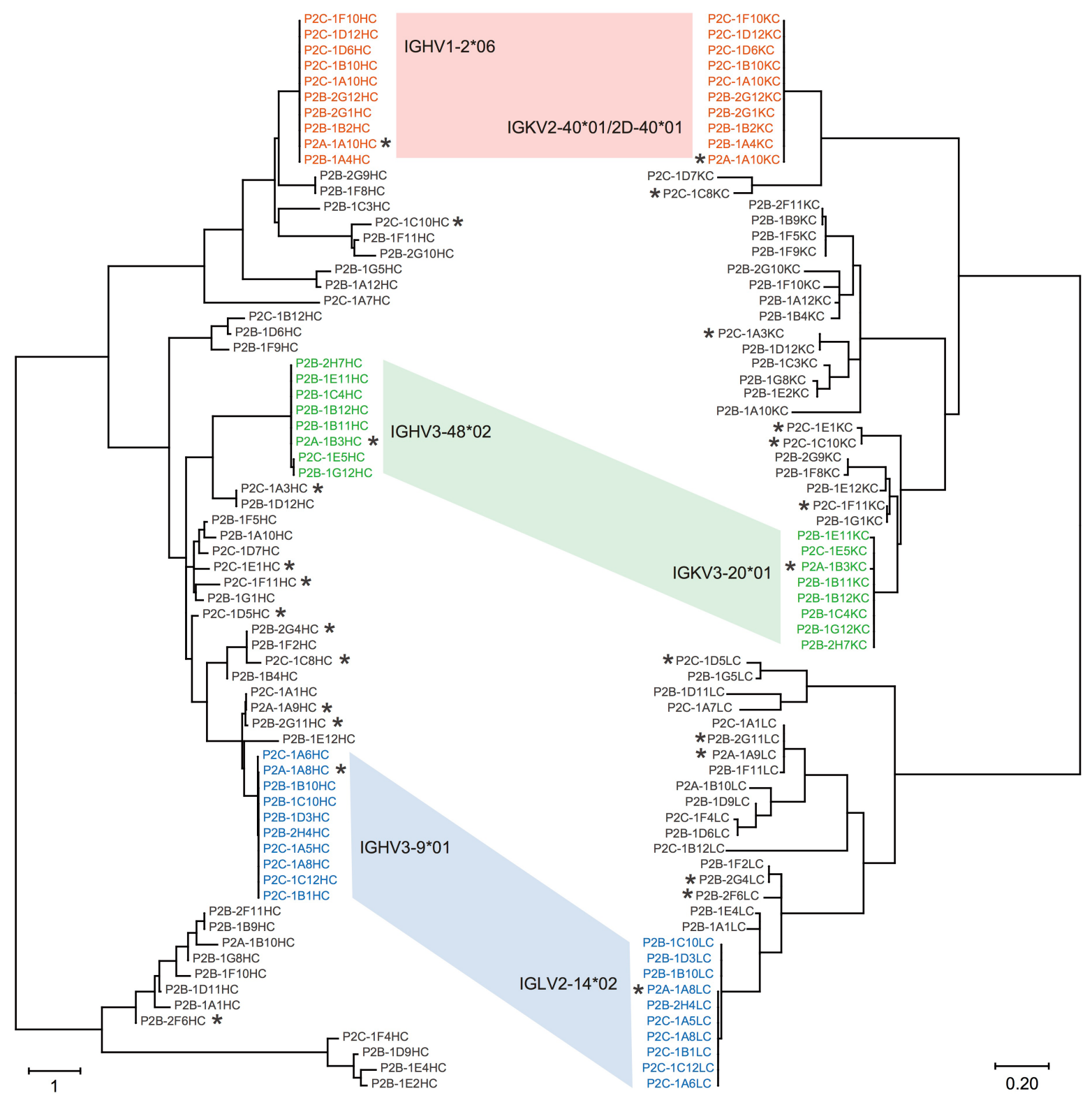

Extended Data Fig. 4 | Genetic analysis. Phylogenetic analysis of heavy (left) colours. Branch lengths are drawn to scale so that sequence relatedness can be and light (right) genes for all RBD-binding antibodies from patient no. 2. Clonal expanded heavy and light clusters are paired and highlighted in three different readily assessed. 
Patient \#2

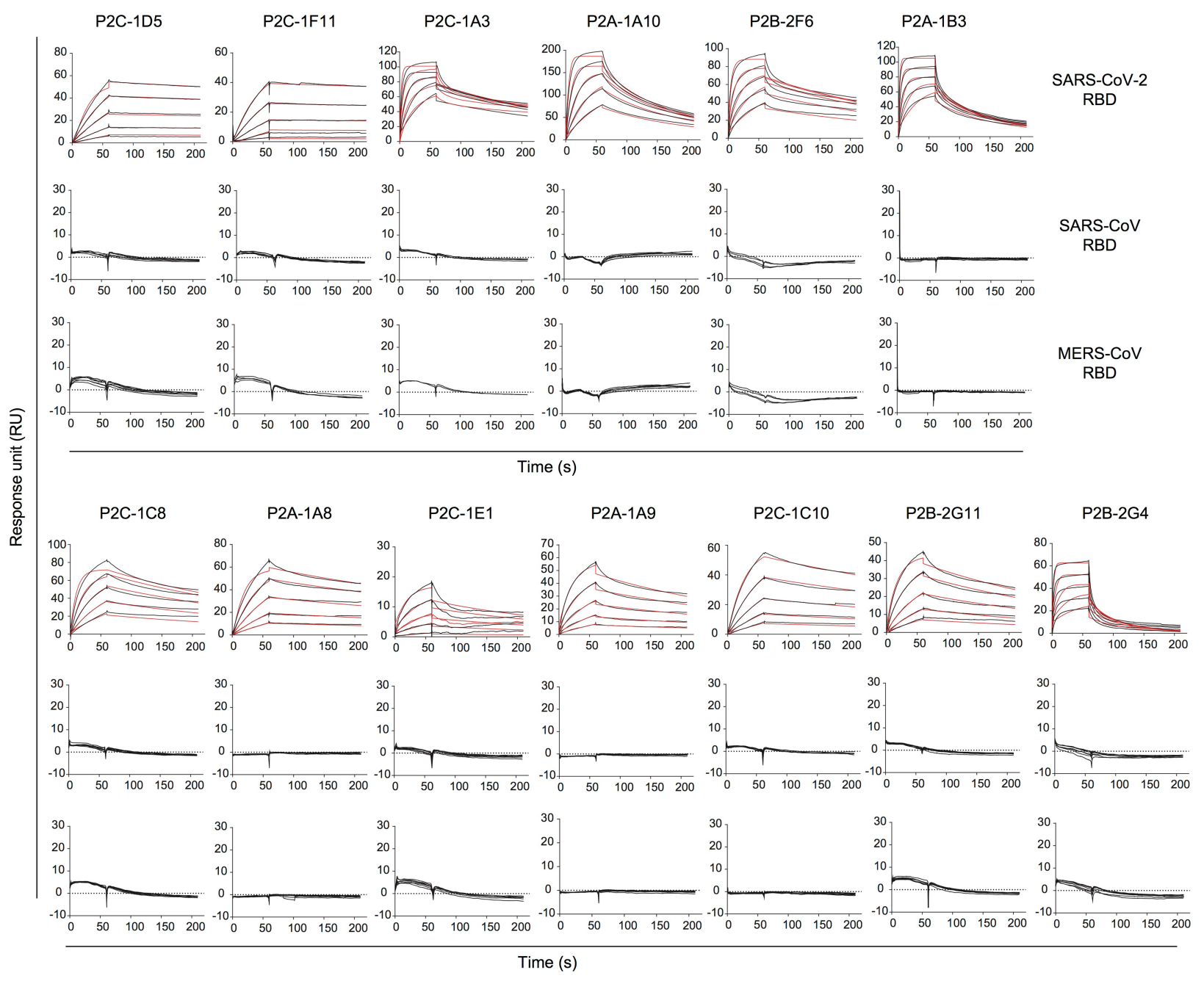

Patient \#1

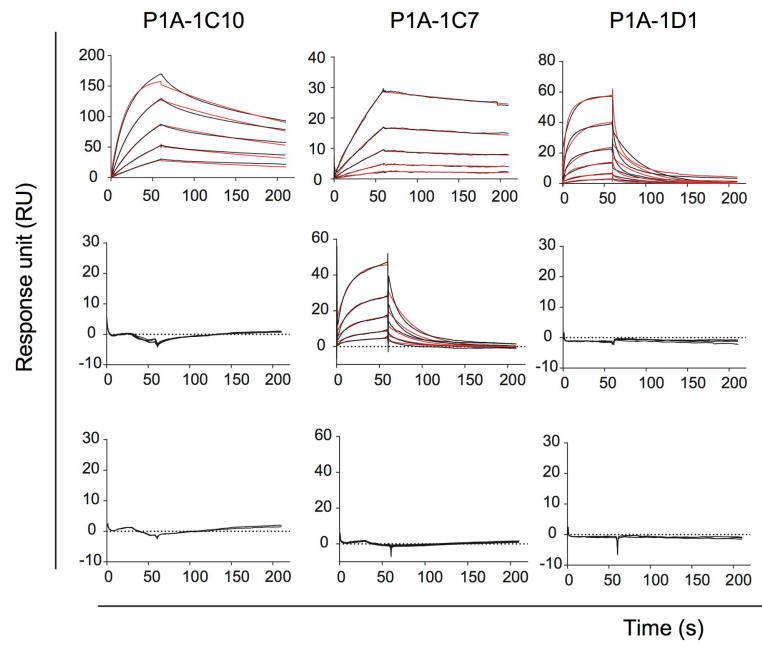

Extended Data Fig. 5 | Binding kinetics of isolated monoclonal antibodies with SARS-CoV-2 RBD measured by SPR. The purified soluble SARS-CoV-2 RBD were covalently immobilized onto a CM5 sensor chip followed by injection of individual antibody at five different concentrations. The black lines indicate
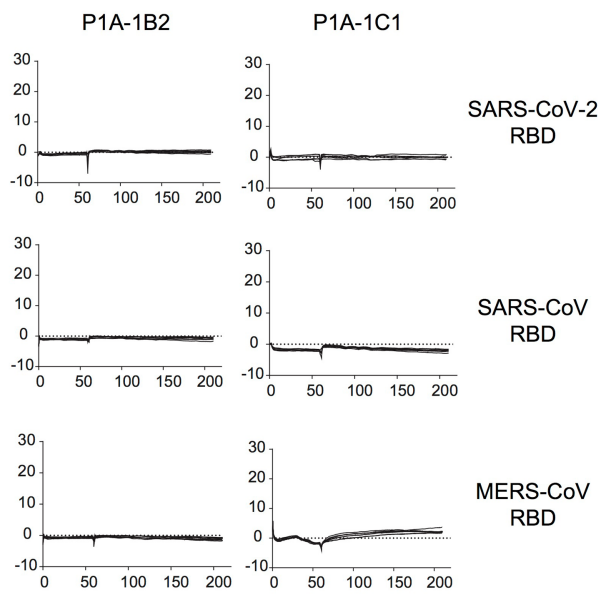

the experimentally derived curves while the red lines represent fitted curves based on the experimental data. Results presented are representatives of two independent experiments. 
Patient \#2
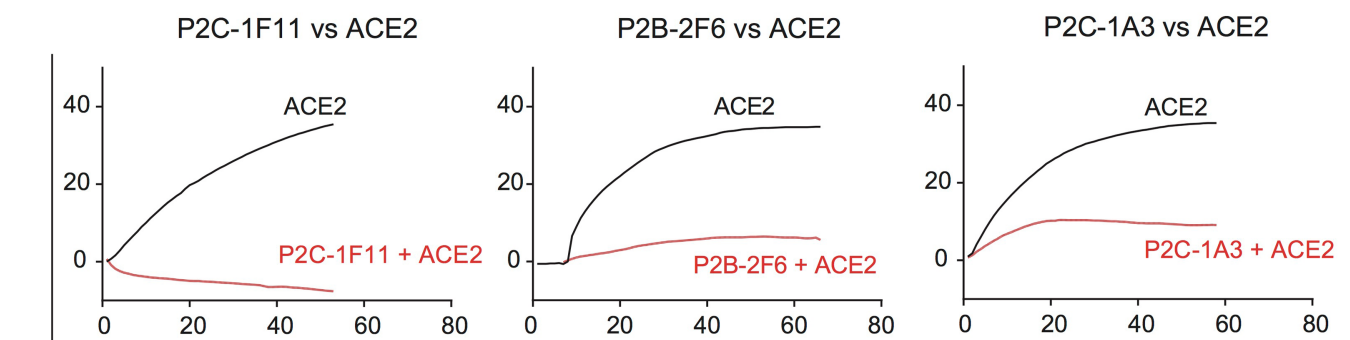

$\mathrm{P} 2 \mathrm{~A}-1 \mathrm{~A} 10$ vs ACE2

P2C-1C8 vs ACE2

P2C-1C10 vs ACE2
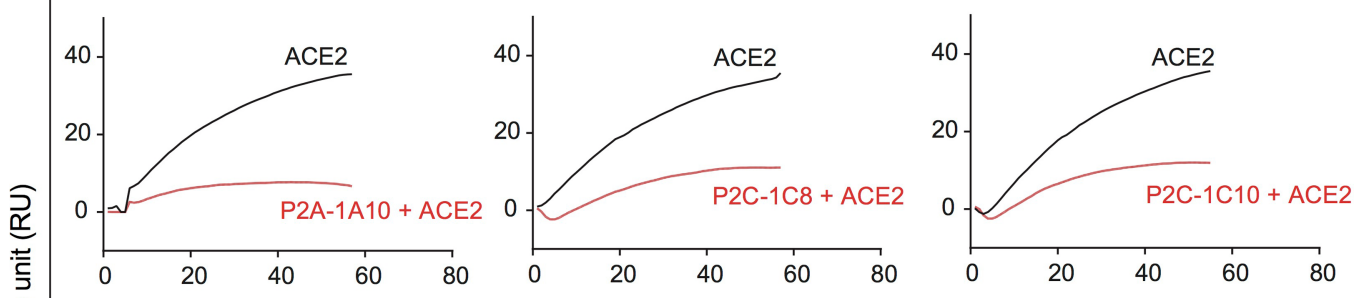

ญ

P2A-1B3 vs ACE2

P2A-1A8 vs ACE2

P2C-1E1 vs ACE2
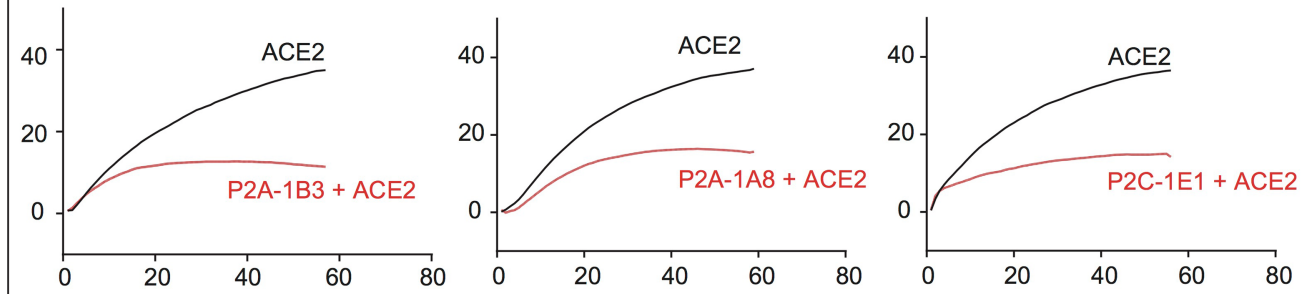

P2A-1A9 vs ACE2

P2B-2G11 vs ACE2

P2B-2G4 vs ACE2

P2C-1D5 vs ACE2
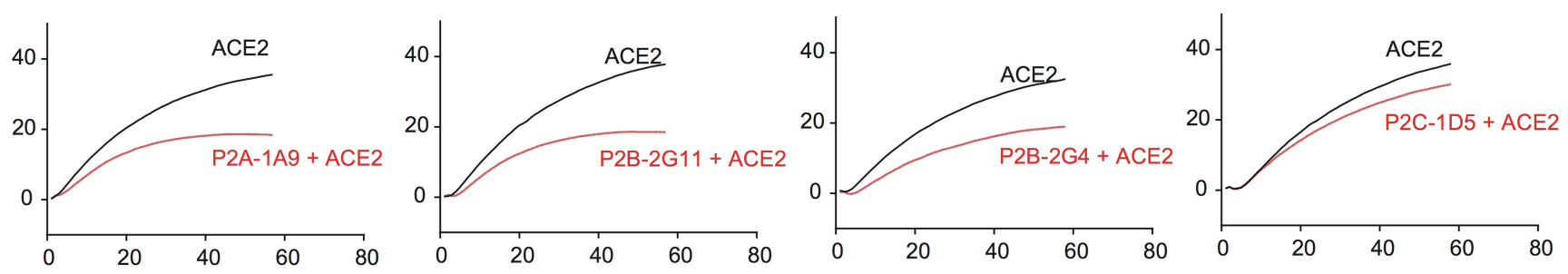

Time (s)

Patient \#1
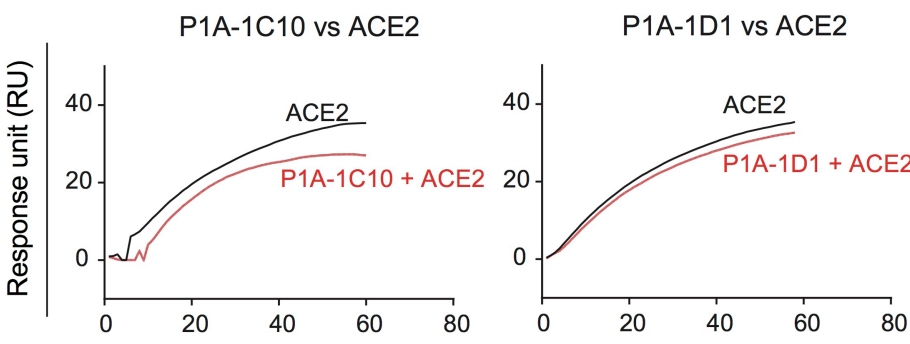

Time (s)

Extended Data Fig. 6 | Antibody and ACE2 competition for binding to SARS-CoV-2 RBD measured by SPR. The sensorgrams show distinct binding patterns of ACE2 to SARS-CoV-2 RBD with (red curve) or without (black curve) prior incubation with each testing antibody. The competition capacity of each antibody is indicated by the level of reduction in response unit of ACE2 comparing with or without prior antibody incubation. Results presented are representatives of two independent experiments. 

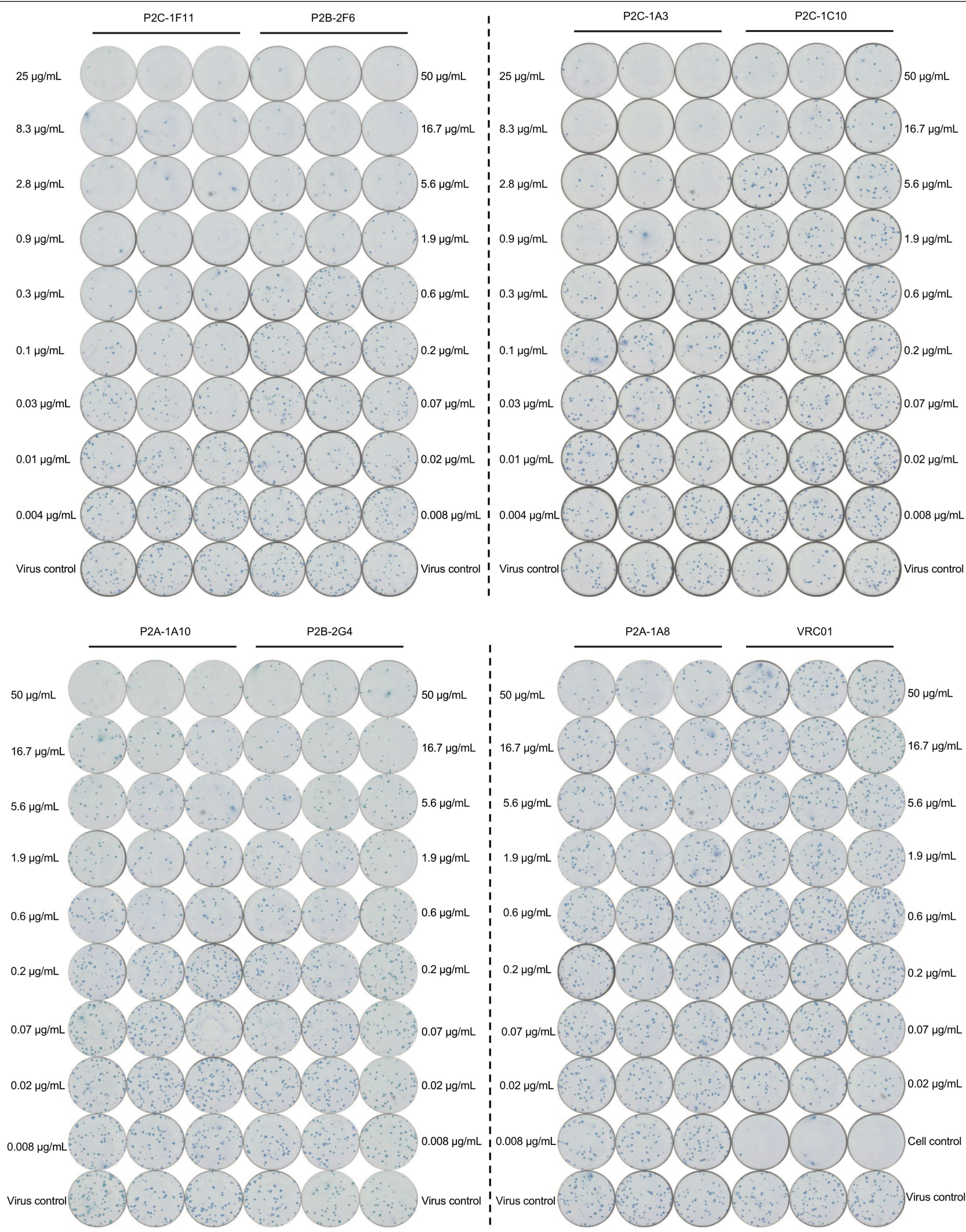

Extended Data Fig. 7 |Neutralization activity of monoclonal antibodies against live SARS-CoV-2 analysed by FRNT. Serial dilution of each antibody was tested against live SARS-CoV-2 infection. Their neutralizing activities are

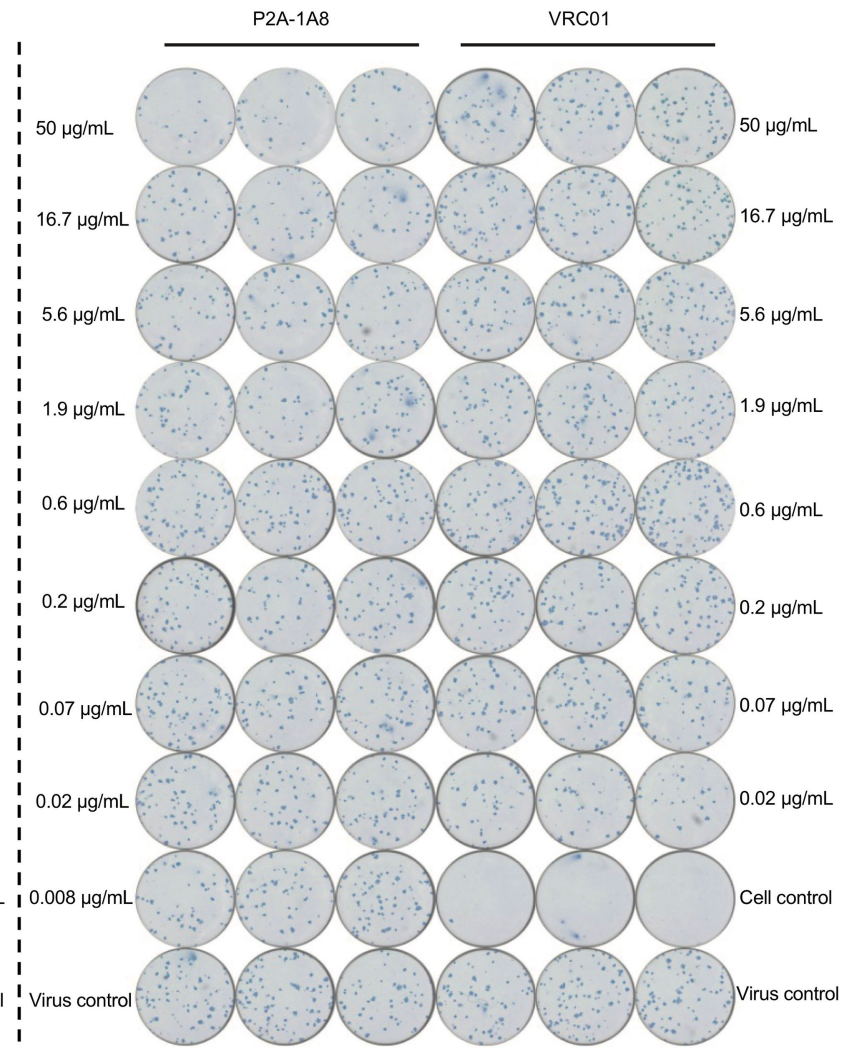

represented by the reduction in the number of SARS-CoV- 2 foci calculated by an EliSpot reader (Cellular Technology). Results $(n=3)$ are representatives of two independent experiments. 

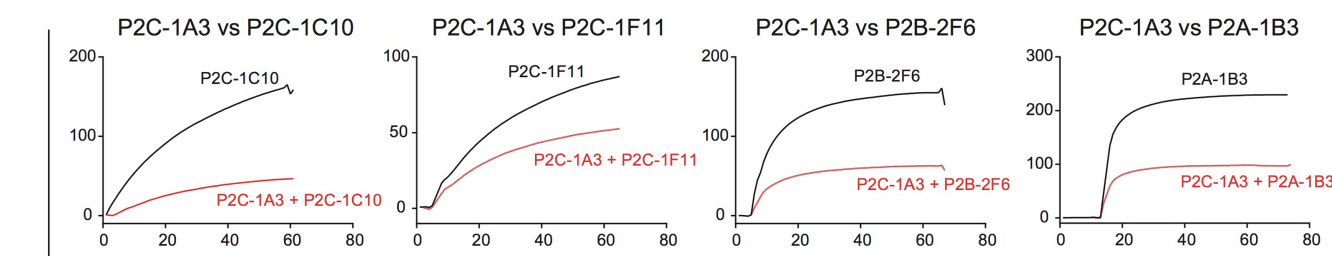

P2C-1A3 vs P2A-1A10

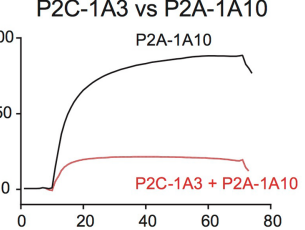

$\mathrm{P} 2 \mathrm{C}-1 \mathrm{C} 10$ vs $\mathrm{P} 2 \mathrm{C}-1 \mathrm{~A} 3$

$\mathrm{P} 2 \mathrm{C}-1 \mathrm{C} 10$ vs $\mathrm{P} 2 \mathrm{C}-1 \mathrm{~F} 11$

$\mathrm{P} 2 \mathrm{C}-1 \mathrm{C} 10$ vs $\mathrm{P} 2 \mathrm{~B}-2 \mathrm{~F} 6$
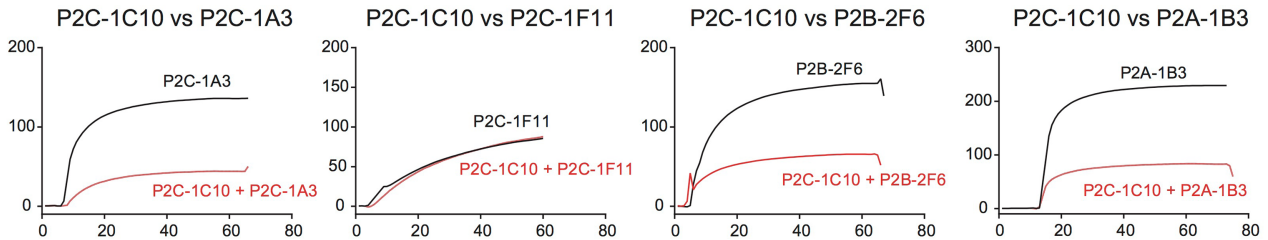

$\mathrm{P} 2 \mathrm{C}-1 \mathrm{C} 10$ vs $\mathrm{P} 2 \mathrm{~A}-1 \mathrm{~A} 10$
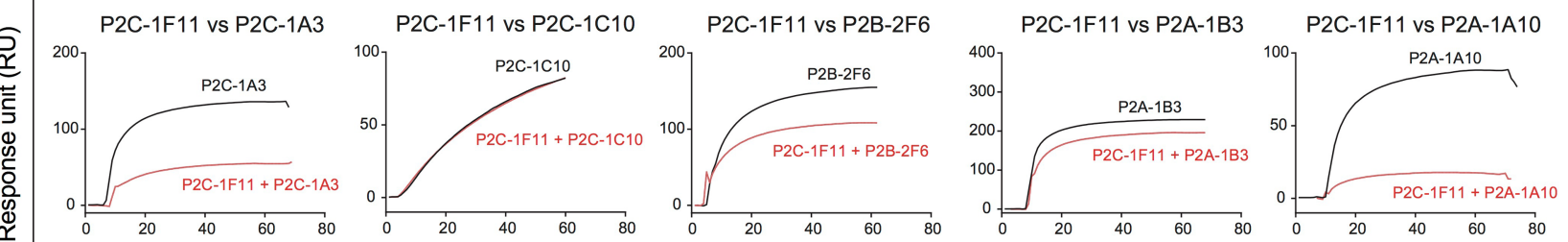

P2B-2F6 vs P2C-1A3
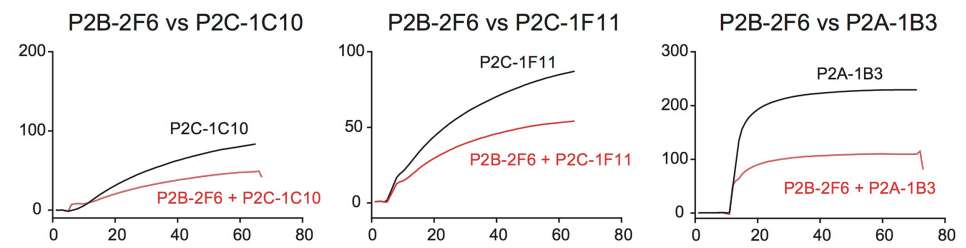

P2B-2F6 vs P2A-1A10

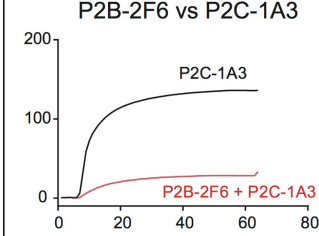

$\mathrm{P} 2 \mathrm{~A}-1 \mathrm{~B} 3$ vs $\mathrm{P} 2 \mathrm{C}-1 \mathrm{~A} 3$

P2A-1B3 vs $\mathrm{P} 2 \mathrm{C}-1 \mathrm{C} 10$

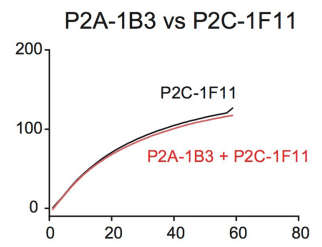

P2A-1B3 vs P2B-2F6
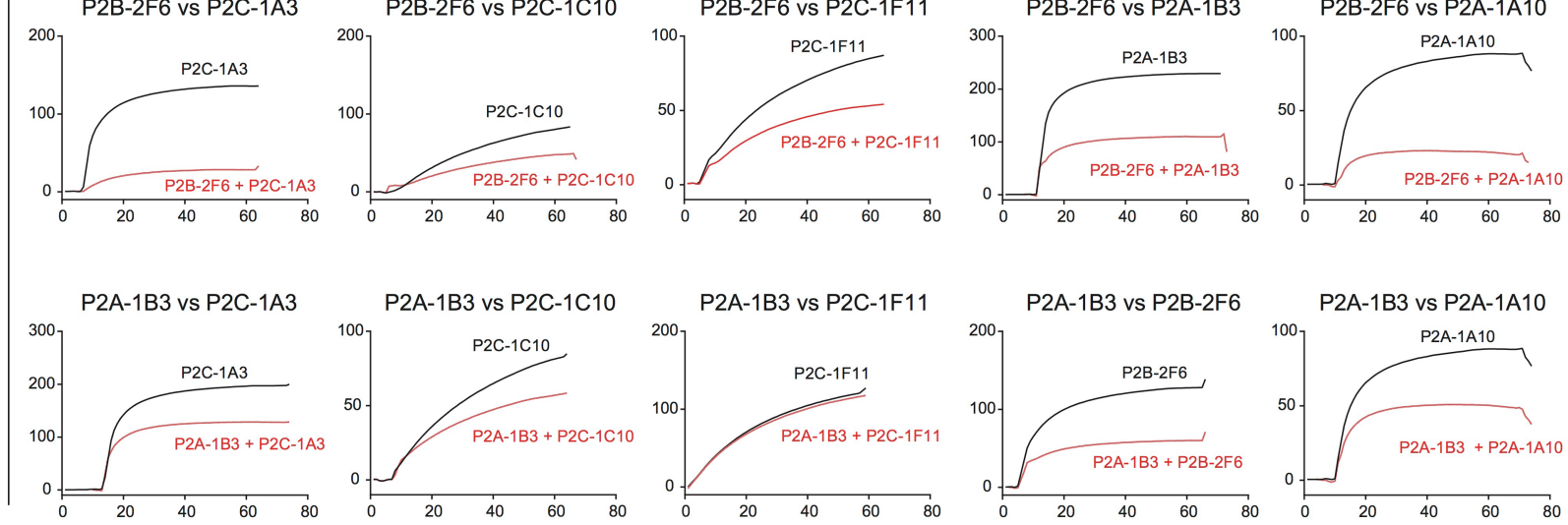

$\mathrm{P} 2 \mathrm{~A}-1 \mathrm{~B} 3$ vs $\mathrm{P} 2 \mathrm{~A}-1 \mathrm{~A} 10$

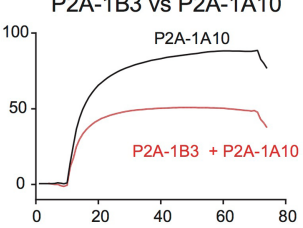

Time (s)

b

\begin{tabular}{lcccccc}
\hline mAbs & P2C-1A3 & P2C-1C10 & P2C-1F11 & P2B-2F6 & P2A-1B3 & P2A-1A10 \\
\hline P2C-1A3 & & ++ & ++ & ++ & ++ & n.a. \\
P2C-1C10 & ++ & & - & + & + & n.a. \\
P2C-1F11 & ++ & - & & + & - & n.a. \\
P2B-2F6 & ++ & ++ & + & & ++ & n.a. \\
P2A-1B3 & ++ & ++ & - & ++ & & n.a. \\
P2A-1A10 & ++ & ++ & ++ & ++ & + & \\
\hline
\end{tabular}

Extended Data Fig. 8 | Epitope mapping through competitive binding measured by SPR. a, The sensorgrams show distinct binding patterns when pairs of testing antibodies were sequentially applied to the purified SARS-CoV-2 RBD covalently immobilized onto a CM5 sensor chip. The level of reduction in response unit comparing with or without prior antibody incubation is the key criterion for determining the two monoclonal antibodies recognize the separate or closely situated epitopes. b, Summary of abovementioned competition in a, in which ' +++ ' indicates $>80 \%$ competition; ' ++ ' 50-80\%; '+' 20-50\%; and '-' $<20 \%$. Results in a are representatives of two independent experiments. n. a.: not applicable. 
Extended Data Table 1 | Binding capacity, neutralizing activity, and gene family analysis of 18 monoclonal Abs isolated from patient no. 1 and patient no. 2

\begin{tabular}{|c|c|c|c|c|c|c|c|c|c|c|c|c|c|}
\hline \multirow{2}{*}{ Patient } & \multirow{2}{*}{ mAbs } & \multicolumn{2}{|c|}{ Binding to RBD } & \multicolumn{2}{|c|}{ Pseudovirus $(\mu \mathrm{g} / \mathrm{ml})$} & \multicolumn{2}{|c|}{ Live virus $(\mu \mathrm{g} / \mathrm{ml})$} & \multicolumn{3}{|c|}{ Heavy chain } & \multicolumn{3}{|c|}{ Kappa chain (K) or Lambda chain (L) } \\
\hline & & $\mathrm{Kd}(\mathrm{nM})$ & competing w/ ACE2 & $\mathrm{IC}_{50}$ & $\mathrm{IC}_{80}$ & $\mathrm{IC}_{50}$ & $\mathrm{I} \mathrm{C}_{80}$ & IGHV & CDR3 length & SHM (\%) & IGK(L)V & CDR3 length & SHM (\%) \\
\hline $\mathrm{P \# 2}$ & $\mathrm{P} 2 \mathrm{C}-1 \mathrm{~F} 11$ & 2.12 & +++ & 0.03 & 0.12 & 0.03 & 0.18 & $3-66^{*} 01,3-66^{*} 04$ & 11 & 1.75 & K3-20*01 & 8 & 0.00 \\
\hline $\mathrm{P \# 2}$ & P2B-2F6 & 5.14 & +++ & 0.05 & 0.61 & 0.41 & 2.43 & $4-38-2^{*} 02$ & 20 & 0.69 & L2-8*01 & 10 & 0.00 \\
\hline $\mathrm{P \# 2}$ & $\mathrm{P} 2 \mathrm{C}-1 \mathrm{~A} 3$ & 2.47 & +++ & 0.62 & 5.94 & 0.28 & 1.46 & $3-11^{*} 04$ & 12 & 0.00 & K1-9*01 & 9 & 0.00 \\
\hline P\#2 & $\mathrm{P} 2 \mathrm{C}-1 \mathrm{C} 10$ & 15.23 & ++ & 2.62 & 4.64 & 11.12 & $>50$ & $1-69 * 01,1-69 D^{*} 01$ & 11 & 0.35 & $\mathrm{~K} 3-11^{*} 01$ & 8 & 0.00 \\
\hline P\#2 & P2B-2G4 & 21.29 & + & 5.11 & $>50$ & 2.90 & 47.70 & $3-33^{*} 01,3-33^{*} 06$ & 11 & 0.00 & L2-11*01 & 11 & 0.00 \\
\hline P\#2 & $\mathrm{P} 2 \mathrm{~A}-1 \mathrm{~A} 8$ & 8.91 & ++ & 7.68 & 26.41 & 35.87 & $>50$ & $3-9^{*} 01$ & 23 & 3.82 & L2-14*02 & 10 & 2.59 \\
\hline $\mathrm{P \# 2}$ & P2A-1A10 & 4.65 & +++ & 8.57 & 39.44 & 1.64 & 22.15 & $1-2^{*} 06$ & 19 & 0.00 & $\mathrm{~K} 2-40^{*} 01, \mathrm{~K} 2 \mathrm{D}-40^{*} 01$ & 9 & 0.00 \\
\hline P\#2 & P2C-1D5 & 1.38 & - & 10.65 & 25.36 & n.d. & n.d. & $3-23^{*} 04$ & 14 & 0.69 & L3-21*01 & 11 & 0.38 \\
\hline P\#2 & P2A-1B3 & 6.00 & ++ & 16.77 & $>50$ & n.d. & n.d. & $3-48 * 02$ & 16 & 10.07 & $\mathrm{~K} 3-20^{*} 01$ & 10 & 3.00 \\
\hline P\#2 & P2A-1A9 & 15.18 & ++ & 26.27 & $>50$ & n.d. & n.d. & $3-9^{*} 01$ & 17 & 2.08 & L1-40*01 & 11 & 1.11 \\
\hline $\mathrm{P \# 2}$ & P2C-1C8 & 8.76 & ++ & 34.38 & $>50$ & n.d. & n.d. & $3-33^{*} 01,3-33^{*} 06$ & 13 & 0.69 & K2D-30*01 & 9 & 0.36 \\
\hline P\#2 & P2B-2G11 & 17.57 & ++ & 34.84 & $>50$ & n.d. & n.d. & $3-9 * 01$ & 17 & 2.08 & L1-40*01 & 11 & 1.11 \\
\hline $\mathrm{P \# 2}$ & P2C-1E1 & 14.99 & ++ & $>50$ & $>50$ & n.d. & n.d. & $3-66^{*} 01,3-66^{*} 04$ & 9 & 0.00 & K3-11*01 & 10 & 0.00 \\
\hline P\#1 & $\mathrm{P} 1 \mathrm{~A}-1 \mathrm{C} 7$ & 51.08 & - & $>50$ & $>50$ & n.d. & n.d. & $1-46^{\star} 01,1-46^{*} 03$ & 15 & 0.00 & $\mathrm{~K} 1-39^{*} 01, \mathrm{~K} 1 \mathrm{D}-39^{*} 01$ & 10 & 0.00 \\
\hline P\#1 & P1A-1C10 & 8.48 & + & $>50$ & $>50$ & n.d. & n.d. & $1-69 * 09$ & 16 & 10.42 & K1-5*03 & 9 & 3.41 \\
\hline P\#1 & P1A-1D1 & 260.50 & - & $>50$ & $>50$ & n.d. & n.d. & $3-53^{*} 01$ & 12 & 4.21 & L2-8*01 & 10 & 2.22 \\
\hline P\#1 & P1A-1B2 & n.d. & n.d. & n.d. & n.d. & n.d. & n.d. & $3-30 * 03,3-30 * 18,3-30-5^{*} 01$ & 12 & 11.46 & L2-14*01 & 10 & 9.26 \\
\hline P\#1 & P1A-1C1 & n.d. & n.d. & n.d. & n.d. & n.d. & n.d. & $3-33^{*} 01,3-33^{*} 05,3-33^{*} 06$ & 17 & 6.25 & K1D-13*01 & 9 & 5.68 \\
\hline
\end{tabular}

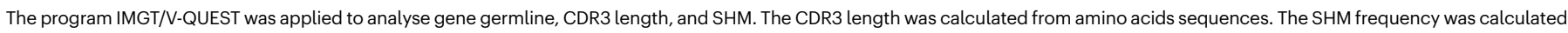
from the mutated nucleotides. Antibody binding to RBD was presented either by $K_{d}$ or by competing with ACE2, in which ' +++ ' indicates $>80 \%$ competition; ' $++{ }^{\prime} 50-80 \%$; ' + ' $20-50 \%$; and ' - ' $<20 \% . I_{50}$ represents the half-maximal whereas $I_{80}$ the $80 \%$ inhibitory concentrations in the pseudovirus and live SARS-CoV-2 neutralization assay. n.d. not done. 


\section{Article}

Extended Data Table 2 | Data collection and refinement statistics

SARS-CoV-2 RBD-P2B-2F6

complex

Data collection

Space group

$\mathrm{P} 22_{1} 2_{1}$

Cell dimensions

$a, b, c(\AA)$

$\alpha, \beta, \gamma\left({ }^{\circ}\right)$

Resolution $(\AA)$

$R_{\text {sym }}$ or $R_{\text {merge }}$

$I / \sigma I$

Completeness (\%)

Redundancy

$70.23,90.15,112.35$

$90,90,90$

56.17-2.85(2.95-2.85) *

$0.154(1.452)$

$14.3(2.2)$

99.80(99.94)

12.9(13.5)

\section{Refinement}

Resolution $(\AA)$

No. reflections

56.17-2.85

17226

$R_{\text {work }} / R_{\text {free }}$

$21.9 / 26.0$

No. atoms

Protein

Ligand/ion

4805

14

$B$-factors

Protein

74.46

Ligand/ion

84.78

R.m.s. deviations Bond lengths $(\AA)$

0.008

Bond angles $\left({ }^{\circ}\right)$

1.04

*One crystal for the data

*Values in parentheses are for highest-resolution shell. 


\section{Reporting Summary}

Nature Research wishes to improve the reproducibility of the work that we publish. This form provides structure for consistency and transparency in reporting. For further information on Nature Research policies, seeAuthors \& Referees and theEditorial Policy Checklist .

\section{Statistics}

For all statistical analyses, confirm that the following items are present in the figure legend, table legend, main text, or Methods section.

n/a $\mid$ Confirmed

$\square \quad \boldsymbol{x}$ The exact sample size ( $n$ ) for each experimental group/condition, given as a discrete number and unit of measurement

$\square \times$ A statement on whether measurements were taken from distinct samples or whether the same sample was measured repeatedly

$\mathbf{x} \square$ The statistical test(s) used AND whether they are one- or two-sided

$x \square$ Only common tests should be described solely by name; describe more complex techniques in the Methods section.

$\mathbf{x} \square$ A description of all covariates tested

$\boldsymbol{x} \square$ A description of any assumptions or corrections, such as tests of normality and adjustment for multiple comparisons

x $\square$ A full description of the statistical parameters including central tendency (e.g. means) or other basic estimates (e.g. regression coefficient)

$\boldsymbol{x} \square$ AND variation (e.g. standard deviation) or associated estimates of uncertainty (e.g. confidence intervals)

$\mathbf{x} \square$ For null hypothesis testing, the test statistic (e.g. $F, t, r$ ) with confidence intervals, effect sizes, degrees of freedom and $P$ value noted

$\boldsymbol{x} \square$ Give $P$ values as exact values whenever suitable.

$\mathbf{x} \square$ For Bayesian analysis, information on the choice of priors and Markov chain Monte Carlo settings

$\boldsymbol{x} \square$ For hierarchical and complex designs, identification of the appropriate level for tests and full reporting of outcomes

$\mathbf{x} \square$ Estimates of effect sizes (e.g. Cohen's $d$, Pearson's $r$ ), indicating how they were calculated

Our web collection on statistics for biologists contains articles on many of the points above.

\section{Software and code}

Policy information about availability of computer code

Data collection Flow cytometry BD Aria II was used for for cell sorting and Biacore T200 Control Software was used for binding kinetic studies.

Data analysis The program IMGT/V-QUEST (http://www.imgt.org/IMGT_vquest/vquest) was applied to analyze gene germline, complementarity determining region (CDR) 3 length, and somatic hypermutation (SHM). The CDR3 length was calculated from amino acids sequences. The SHM frequency was calculated from the mutated nucleotides. Graphs were presented by GraphPad Prism version 7, R version 3.6.2, MEGA version X, and BIAcore T200 Evaluation version 3.1 softwares. Flow cytometry data analysis was performed using FlowJo version 10 software.

For manuscripts utilizing custom algorithms or software that are central to the research but not yet described in published literature, software must be made available to editors/reviewers. We strongly encourage code deposition in a community repository (e.g. GitHub). See the Nature Research guidelines for submitting code \& software for further information.

\section{Data}

Policy information about availability of data

All manuscripts must include a data availability statement. This statement should provide the following information, where applicable:

- Accession codes, unique identifiers, or web links for publicly available datasets

- A list of figures that have associated raw data

- A description of any restrictions on data availability

Crystal structure presented in this work has been deposited in the Protein Data Bank (PDB) and are available with accession codes 7BWJ. Other data generated or analyzed during this study are included in this published article (and its supplementary information files). Any other raw data pertaining to this stu dy are available from the corresponding authors upon reasonable request. 


\section{Field-specific reporting}

Please select the one below that is the best fit for your research. If you are not sure, read the appropriate sections before making your selection.
$\boldsymbol{x}$ Life sciences
Behavioural \& social sciences
Ecological, evolutionary \& environmental sciences

For a reference copy of the document with all sections, see nature.com/documents/nr-reporting-summary-flat.pdf

\section{Life sciences study design}

All studies must disclose on these points even when the disclosure is negative. $\begin{array}{ll}\text { Sample size } & \text { We have isolated antibodies from PBMCs of eight SARS-CoV-2-infected donors, including } 3 \text { severe patients and } 5 \text { mild patients, aging from } 10 \\ \text { to } 66 \text { years old. This sample size is sufficient for isolating neutralizing antibodies. }\end{array}$

Data exclusions No data were excluded.

Replication ELISA analysis, neutralization assay, epitope mapping experiments, mabs binding to cell surface expressed spike proteins, the crystal of complex were peformed in duplicate. All attempts at replication were successful.

Randomization Not applicable for this study as no treatment strategies are compared.

Blinding No blinding was conducted since there was no specific grouping.

\section{Reporting for specific materials, systems and methods}

We require information from authors about some types of materials, experimental systems and methods used in many studies. Here, indicate whether each material, system or method listed is relevant to your study. If you are not sure if a list item applies to your research, read the appropriate section before selecting a response.

\begin{tabular}{|c|c|c|}
\hline$n / a$ & & olved in the study \\
\hline & $x$ & Antibodies \\
\hline & $x$ & Eukaryotic cell lines \\
\hline$x$ & & Palaeontology \\
\hline$x$ & 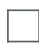 & Animals and other organisms \\
\hline$\square$ & $x$ & Human research participants \\
\hline$x$ & & Clinical data \\
\hline
\end{tabular}

\begin{tabular}{l|l}
\multicolumn{2}{l}{ Methods } \\
\hline n/a & Involved in the study \\
\hline $\boldsymbol{x}$ & $\square$ ChIP-seq \\
\hline & $\boldsymbol{x}$ Flow cytometry \\
\hline $\boldsymbol{x}$ & $\square$ MRI-based neuroimaging
\end{tabular}

\section{Antibodies}

Antibodies used

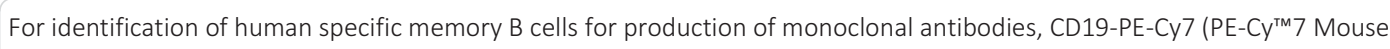
Anti-Human CD19, BD Pharmingen, cat. 557835, clone SJ25C1, lot. 8194923, 1:50 dilution), CD3-Pacific Blue (Pacific Blue ${ }^{\mathrm{TM}}$ Mouse Anti-Human CD3, BD Pharmingen, cat. 558117, clone UCHT1, lot. 8183535, 1:50 dilution), CD8-Pacific Blue (Pacific Blue ${ }^{\mathrm{TM}}$ Mouse Anti-Human CD8, BD Pharmingen, cat. 558207, clone RPA-T8, lot.8127596, 1:25 dilution), CD14-Pacific Blue (Pacific Blue ${ }^{\text {TM }}$ Mouse Anti-Human CD14, BD Pharmingen, cat. 558121, clone M5E2, lot. 7164513, 1:50 dilution), CD27-APC-H7 (APC-H7 Mouse Anti-Human CD27, BD Pharmingen, cat. 560222, clone M-T271, lot. 8256900, 1:25 dilution), IgG-FITC (FITC Mouse Anti-Human IgG, BD Pharmingen, cat. 555786, clone G18-145, lot. 8284569, 1:12.5 dilution), anti-his-APC (Anti-6X His tag ${ }^{\circledR}$ antibody SureLight ${ }^{\circledR}$ Allophycocyanin, Abcam, cat. ab72579, clone AD1.1.10, lot. GR3192034-1, 1:25 dilution) and anti-his-PE (Anti-6X His tag $^{\circledR}$ antibody Phycoerythrin, Abcam, cat. ab72467, clone AD1.1.10, lot. GR3223742-7, 1:25 dilution) antibodies were used. For characterization of human antibodies, secondary anti-human IgG-HRP (HRP goat anti-human IgG (H+L), ZSGB-BIO, cat. ZB-2304, polyclonal, lot. 118693, 1:5000 dilution) and anti-human Fc-PE (PE anti-human IgG Fc, Biolegend, cat. 410708, clone M1310G05, lot. B305154, 1:20 dilution) antibodies were used.

Validation

All the antibodies used in this study were commercial antibodies and were only used for applications, with validation procedures described on the following sites of the manufacturers:

CD19-PE-Cy7 (PE-CY'TM7 Mouse Anti-Human CD19, BD Pharmingen, cat. 557835, clone SJ25C1, lot. 8194923, 1:50 dilution) https://www.bdbiosciences.com/cn/applications/research/clinical-research/oncology-research/blood-cell-disorders/surfacemarkers/human/pe-cy7-mouse-anti-human-cd19-sj25c1-also-known-as-sj25-c1/p/557835

CD3-Pacific Blue (Pacific Blue ${ }^{\mathrm{TM}}$ Mouse Anti-Human CD3, BD Pharmingen, cat. 558117, clone UCHT1, lot. 8183535, 1:50 dilution) https://www.bdbiosciences.com/cn/applications/research/t-cell-immunology/th-1-cells/surface-markers/human/pacific-bluemouse-anti-human-cd3-ucht1-also-known-as-ucht-1-ucht-1/p/558117

CD8-Pacific Blue (Pacific Blue ${ }^{\mathrm{TM}}$ Mouse Anti-Human CD8, BD Pharmingen, cat. 558207, clone RPA-T8, lot.8127596, 1:25 dilution) https://www.bdbiosciences.com/cn/reagents/research/antibodies-buffers/immunology-reagents/anti-human-antibodies/cell- 
surface-antigens/pacific-blue-mouse-anti-human-cd8-rpa-t8/p/558207

CD14-Pacific Blue (Pacific Blue ${ }^{\mathrm{TM}}$ Mouse Anti-Human CD14, BD Pharmingen, cat. 558121, clone M5E2, lot. 7164513, 1:50 dilution) https://www.bdbiosciences.com/cn/applications/research/stem-cell-research/hematopoietic-stem-cell-markers/human/ negative-markers/pacific-blue-mouse-anti-human-cd14-m5e2/p/558121

CD27-APC-H7 (APC-H7 Mouse Anti-Human CD27, BD Pharmingen, cat. 560222, clone M-T271, lot. 8256900, 1:25 dilution) https://www.bdbiosciences.com/cn/applications/research/clinical-research/oncology-research/blood-cell-disorders/surfacemarkers/human/apc-h7-mouse-anti-human-cd27-m-t271/p/560222

IgG-FITC (FITC Mouse Anti-Human IgG, BD Pharmingen, cat. 555786, clone G18-145, lot. 8284569, 1:12.5 dilution)

https://www.bdbiosciences.com/cn/applications/research/b-cell-research/immunoglobulins/human/fitc-mouse-anti-human-iggg18-145/p/555786

anti-his-APC (Anti-6X His tag ${ }^{\circledR}$ antibody SureLight ${ }^{\circledR}$ Allophycocyanin, Abcam, cat. ab72579, clone AD1.1.10, lot. GR3192034-1, 1:25 dilution)

https://www.abcam.com/6x-his-tag-antibody-ad1110-surelight-allophycocyanin-ab72579.html

anti-his-PE (Anti-6X His tag ${ }^{\circledR}$ antibody Phycoerythrin, Abcam, cat. ab72467, clone AD1.1.10, lot. GR3223742-7, 1:25 dilution)

https://www.abcam.com/6x-his-tag-antibody-ad1110-phycoerythrin-ab72467.html

secondary anti-human IgG-HRP (HRP goat anti-human IgG (H+L) , ZSGB-BIO, cat. ZB-2304, polyclonal, lot. 118693, 1:5000 dilution)

http://www.zsbio.com/product/zb-2304

anti-human Fc-PE (PE anti-human IgG Fc, Biolegend, cat. 410708, clone M1310G05, lot. B305154, 1:20 dilution)

https://www.labome.com/product/BioLegend/410708.html

\section{Eukaryotic cell lines}

Policy information about cell lines

Cell line source(s)

The 293 T cells, Sf9 cells, Hi5 cells, ghost cells and Huh7 cells were obtained from ATCC. The 293F cells were purchased from ThermoFisher.

Authentication

All cell lines were frequently checked for cellular morphologies, growth rates and functions. All cell lines were available in commercial company.

Mycoplasma contamination

We confirm that all cell lines were negative for mycoplasma contamination.

Commonly misidentified lines

(See ICLAC register)

No commonly misidentified cell lines were used.

\section{Human research participants}

Policy information about studies involving human research participants

Population characteristics The study enrolled a total of eight patients aged 10 to 66 years old infected with SARS-CoV-2 in January 2020.

Recruitment Study participants were recruited on the random basis from COVID-19 confirmed cases, including 3 severe patients and 5 mild patients. There was no potential self-selection bias or other biases during the selection.

Ethics oversight

This study received approval from the Research Ethics Committee of Shenzhen Third People's Hospital, China (approval number: 2020-084). The Research Ethics Committee waived the requirement informed consent before the study started because of the urgent need to collect epidemiological and clinical data. We analyzed all the data anonymously.

Note that full information on the approval of the study protocol must also be provided in the manuscript.

\section{Flow Cytometry}

Plots

Confirm that:

$\boldsymbol{x}$ The axis labels state the marker and fluorochrome used (e.g. CD4-FITC).

$\boldsymbol{x}$ The axis scales are clearly visible. Include numbers along axes only for bottom left plot of group (a 'group' is an analysis of identical markers).

$\boldsymbol{x}$ All plots are contour plots with outliers or pseudocolor plots.

$\square$ A numerical value for number of cells or percentage (with statistics) is provided.

\section{Methodology}

Sample preparation

PBMCs from infected individuals were collected and incubated with an antibody and RBD cocktail for identification of RBDspecific B cells. The cocktail consisted of CD19-PE-Cy7, CD3-Pacific Blue, CD8-Pacific Blue, CD14-Pacific Blue, CD27-APC-H7, IgGFITC (BD Biosciences) and the recombinant RBD-Strep or RBD-His. Three consecutive staining steps were conducted. The first

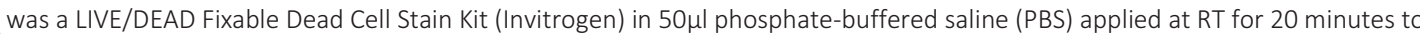


exclude dead cells. The second utilized en antibody and RBD cocktail for an additional 30 mir at $4^{\circ} \mathrm{C}$. The third staining at $4{ }^{\circ} \mathrm{C}$ for 30min involved either: Streptavidin-APC (eBioscience) and/or Streptavidin-PE (BD Biosciences) to target the Strep tag of RBD, or anti-his-APC and anti-his-PE antibodies (Abcam) to target the His tag of RBD. The stained cells were washed and resuspended in PBS before being strained through a 70 um cell mesh (BD Biosciences). More information available or Methods sections.

Instrument

\section{Software}

Cell population abundance

Gating strategy

\section{BD Aria II}

FlowJo version $1 \mathrm{C}$

The RBD-specific $B$ cells constitute about $0.092 \%-1.86 \%$ among the $C D 27+\lg G+B$ cell population. More Information available cn Figure $2 \hat{c}$ and Extended Data Figure 2.

RBD-specific B cells were gated as CD19+CD3-CD8-CD14-IgG+RBD+. More Information available on Figure 2a, Extended Data Figure 2, and Methods sections.

$\boldsymbol{x}$ Tick this box to confirm that a figure exemplifying the gating strategy is provided in the Supplementary Information. 